\title{
Design and Implementation of a Most Secure Cryptographic Scheme for Lightweight Environment using Elliptic Curve and Trigonohash Technique
}

\author{
BhaskarPrakash Kosta ${ }^{1}$, Dr. PasalaSanyasi Naidu ${ }^{2}$ \\ Computer Science and Engineering Department \\ GIT, GITAM Deemed to be University \\ Vishakhapatnam, AP, India
}

\begin{abstract}
The Internet of Things (IoT) is a rising development and is an organization of all gadgets that can be gotten to through the web. As a central advancement of the IoT, wireless sensor networks (WSN) can be used to accumulate the vital environment parameters for express applications. In light of the resource limitation of sensor devices and the open idea of remote channel, security has become an enormous test in WSN. Validation as an essential security service can be used to guarantee the authenticity of data access in WSN. The proposed three factor system using one way hash function is depicted by low computational cost, and limit overhead, while achieving all other benefits. Keys are made from secret key for meeting for improving the security. We differentiated the arrangement's security and execution with some lightweight plans. As shown by the examination, the proposed plan can give more prominent security incorporates low overhead of correspondence. Encryption and unscrambling is done using numerical thoughts and by using the possibility of hash function. Mathematical thoughts are lightweight and update the security up by a staggering degree by diminishing the chances of cryptanalysis. When contrasted with different calculations, the proposed calculation gives better execution results.
\end{abstract}

Keywords-Internet of Things (IoT); authentication; one way hash function; lightweight environment; secret key

\section{INTRODUCTION}

As far off correspondence headway has made, brilliant world individuals can work by utilizing impending Internet of Thing (IoT) paradigm[1]. The fundamental contemplated the Internet of things, or IoT, is a strategy of interrelated getting ready contraptions, mechanical and automated machines, things, creatures or individuals that are given great identifiers (UIDs) and the capacity to move information over an association without expecting that human should human or human-to-PC facilitated exertion. Numerous splendid application can be made by using the data delivered by radiofrequency identification(RFID) and remote sensor organization. A comparable idea can be applied in various mechanical field[2] where canny application can be made using IoT devices. For example in healthcare[3] some distinguishing devices or sensors are embedded or attached to customer which assemble some basic information about customer, for instance, ECG, pulse, temperature and blood oxygen. these fundamental information is then shipped off affirmed clinical master with the help of a section encouraged by the affiliation who runs the center, taking into account which the treatment is overseen without the actual presence of clinical master (This technique can be used to perceive COVID-19 patient among the people who are in separate so the spread of sickness can be avoided).

Using WSN biological data can be accumulated. WSN are regularly involved customers, passages(gateway server) and sensors centers. By and large sensor centers have limited energy, computational force and limit power moreover it has been seen that the energy usage of sensor center depends upon correspondence detachment [4][28], the energy usage for sending or tolerating a message of $l$-bits over or from a distance (d) are assessed by Eqs. 1 and 2, independently. Here the free space model is used if (d) isn't by and large a cutoff $\mathrm{d} 0$; something different, multi-way model is used.

$E_{r}(l, d)=\left\{\begin{array}{l}l E_{\text {elec }}+l \varepsilon_{f s} d^{2} \text { ford }<d_{0} \\ l E_{\text {elec }}+l \varepsilon_{m p} d^{4} \text { ford } \geq d_{0}\end{array}\right.$

$E_{R}(l)=I E_{\text {elec }}$

where $E_{\text {elec }}$ is the energy required by the electronic circuit, $\varepsilon_{f s}$ and $\varepsilon_{m p}$ are the energy required by the amplifier in free space and multi-path model, respectively. One of the plan for correspondence in WSN is showed up in Fig. 1

In Fig. 1, the customer can start the correspondence by sending login message to the gateway(the customer is currently enrolled with the portal the passage(server) moreover keeps up its correspondence with different sensors), as of now the server examinations the sales of customer and makes correspondence with remarkable sensor to make the correspondence possible among customer and sensor. Using this philosophy the power use of sensor center point can be decreased and the life of WSN can be extended. Considering limited security highlight of remote channel and confined resources of IoT devices, IoT faces veritable security and assurance challenges [5].

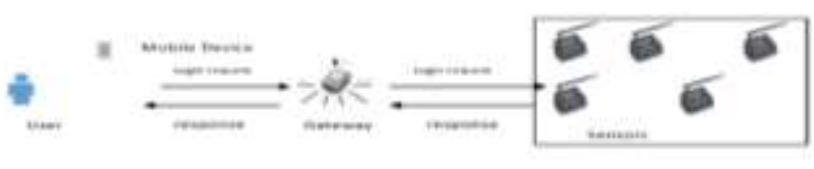

Fig. 1. Architecture for Communication. 
Das in 2009 Das arranged a check scheme[6] for WSN, this arrangement was found having issue with inside attack, impersonation attack and server(gateway) center bypassing attack. In 2012 A.K Das [7] arranged powerful customer approval for progressive WSN using light weight calculation, for instance, hash work and symmetric cryptography, in any case it moreover had issues, for instance, inside attack, server primary key revelation attack[8]. In 2013, Xue et al. [9] proposed a lightweight client validation for WSN, and just hash activities are used to ensure the computational effectiveness. They asserted that their plan is secure against identity/secret key speculating assault and taken smart card assault. Be that as it may, their plan was discovered uncertain against stolen smart card assault, insider assault, following assault, and personality speculating assault [10].

As [8] reveals that most symmetric cryptography and hash based protocols are unreliable to user anonymity and smart card security breach assault, thus public key technique can be used like elliptic curve cryptography (ECC) which outfit same security as RSA with more diminutive key size. In 2011, Yeh et al. [11] considered affirmation convention for WSN reliant on ECC. This show had the issue customer namelessness (secrecy) and undetectability. Thereafter, Shi and Gong [12] presented an improved ECC based check convention for WSN. Regardless, these two shows can't give the features of customer namelessness (secrecy) and unrecognizability. In 2016, Jiang et al. [13] proposed an ECC based untraceable affirmation convention for WSN yet had some security defect as pointed by Li et al. [14].

In rest of this paper oversee proposed three-factor customer approval convention using one way hash function and contrasting encryption using one way hash function and disentangling calculation are similarly discussed and security discussion which are showed up in Sections II and III. Section IV oversees comparison of our show with other related show and discussion of security blemishes of Chang and Le's arrangement, Xiong- Li et al. scheme.

\section{Proposed ANonymity Authentication Protocol FOR IIOT}

In this part, we work with a three-factor validation protocol for modern web of things to accomplish client secrecy and oppose cell phone misfortune assault[15]. Here, we receive the fuzzy extractor [16] to handle biometrics data. More noteworthy degrees of safety are accomplished by biometric secret key, like fingerprints, retina examines. Notwithstanding, two biometric perusing are infrequently indistinguishable, despite the fact that they are probably going to be close. Notation used in full text is shown in Table I. Our plan contains four stages, for example initiation, registration, authentication and key agreement, and password change. We delineate these stages as follows.
TABLE I. NOTATIONS

\begin{tabular}{ll}
\hline Notation & \multicolumn{1}{c}{ Description } \\
\hline $\mathrm{U}_{\mathrm{m}}, \mathrm{ID}_{\mathrm{m}}, \mathrm{PW}_{\mathrm{m}}$ & The Mobile Device(User), and the identification and secret Key \\
$\mathrm{GN}, \mathrm{X}_{\mathrm{GN}}$ & ThegatewayServeranditsServersecret key \\
$\mathrm{S}_{\mathrm{s}}, \mathrm{ID}_{\mathrm{s}}$ & Thesensorhubanditsidentification \\
$\mathrm{xx}, \mathrm{XX}$ & Private key and public key Gateway server \\
$\mathrm{bm}, \mathrm{Mm}$ & Private key and public key of Mobile device(User) \\
$\mathrm{X}_{\mathrm{L}-\mathrm{GN}}$ & Shared secret key between Gateway Serverand Sensor hub \\
$\mathrm{PP}$ & A point on the elliptic curve \\
$\mathrm{H} 1(\cdot), \oplus, \odot$ & Thehash,XOR,andXNORoperation \\
$A$ & AnAttacker \\
\hline
\end{tabular}

\section{A. Initiation Phase}

To introduce the framework, Gateway Server picks a single direction hash work $\mathrm{H1}(\cdot)$ which it imparts to every one of the clients. Additionally, it chooses an elliptic curve E dependent on a limited field Fp. From that point forward, Gateway server picks a subgroup GG of E with request huge prime n, and the generator is point PP. At that point Gateway worker produces a private key $\mathrm{xx}$ and figures the relating public key $\mathrm{XX}$, where $\mathrm{xx} \in \mathrm{Z}^{*} \mathrm{n}$ and $\mathrm{XX}=\mathrm{xxPP}$. Finally, GWN stores $\mathrm{xx}$ furtively and distributes the boundaries $\{\mathrm{E}(\mathrm{Fp}), \mathrm{GG}, \mathrm{PP}, \mathrm{XX}\}$.

\section{1) The Gateway Server(GN)}

a) Gateway server chooses a secret $\mathrm{key}(\mathrm{XGN})$ and one way hash function $\mathrm{H} 1(\cdot)$ which it shares with all the users through secured channel.

b) GN chooses an elliptic curve $\mathrm{E}$ dependent on a limited field Fp. From that point forward, GN picks a subgroup $\mathrm{GG}$ of $\mathrm{E}(\mathrm{Fp})$ with request huge prime $\mathrm{n}$, and the generator is point $\mathrm{PP}$.

c) GN produces a private key $\mathrm{xx}$ and figures the comparing public key $\mathrm{XX}$, where $\mathrm{xx} \in \mathrm{Z} * \mathrm{n}$ and $\mathrm{XX}=\mathrm{xxPP}$.

d) GWN stores $\mathrm{xx}$ covertly and distributes the boundaries $\{\mathrm{E}(\mathrm{Fp}), \mathrm{GG}, \mathrm{PP}, \mathrm{XX}\}$.

e) Sensing Devise( $\mathrm{Ss}$ ) RegistrationPhase.

All the sensing hub(Ss) in IoT are registered offline by the $G N$ as follows.

Step SD1. For each device $S_{s}$, the $G N$ chooses a one way hash function $\mathrm{H} 1(\cdot)$ which it shares with all the sensor nodes in secured manner and a unique identity $I D_{s}$ and calculates the corresponding shared secret key $\mathrm{X}_{\mathrm{IDs}-\mathrm{GN}}$ $=\left(\mathrm{IDs} \oplus \mathrm{X}_{\mathrm{GN}}\right)$. 
Step SD2. The $G N$ pre-loads $\left\{I D_{s}, \mathrm{X}_{\mathrm{IDs}-\mathrm{GN}}, H I(\cdot)\right\}$ in the memory of $S_{s}$

The Sensor Node(Ss)

The Server(GN)

Select IDs for Ss

compute $\mathrm{X}_{\mathrm{IDs}-\mathrm{GN}}=\mathrm{X}_{\mathrm{GN}} \oplus$ IDs

$$
I D_{s}, \mathrm{X}_{\mathrm{IDs}-\mathrm{GN}}
$$

Store IDs, $\mathrm{X}_{\mathrm{IDs}-\mathrm{GN}}, \mathrm{H} 1(\cdot)$

\section{B. Registration Phase}

To be associated with the framework as a legitimate client, the accompanying advances ought to be performed between client Um and GN, and afterward Um can get to the sensor information progressively by utilizing his/her cell phone.

Stage 1. A character IDm, a secret word PWm and an irregular number $\mathrm{rm}$ are picked by Um. Then, Um engraves the biometric data $\mathrm{Bio}_{\mathrm{m}}$ on a cell phone with fuzzy extractor [16] and gets $\operatorname{Gen}\left(\mathrm{Bio}_{\mathrm{m}}\right)=(\sigma \mathrm{m}, \tau \mathrm{m})$. At that point $\mathrm{Um}$ ascertains the veiled secret key RPWm $=\mathrm{PWm} \oplus \mathrm{rm}$, (here $\bigoplus$ stands for XOR operation) and submits \{IDm, RPWm, $\sigma \mathrm{m}\}$ to GN for enrollment.

Stage 2. While getting the enrollment demand, GN checks if IDm in the data set. In the event that indeed, Um requested to present another character. Something else, GN figures Am $=\mathrm{H} 1\left(\mathrm{IDm} \oplus \mathrm{X}_{\mathrm{GN}}\right)$ and $\mathrm{Bm}=\mathrm{Am} \oplus\left(\mathrm{RPWm} \oplus \sigma_{m}\right)$, and sends $\{\mathrm{Bm}, \mathrm{XX}\}$ to $\mathrm{Um}$ through a reliable manner.

\section{Authentication Phase}

When Um wants to access the sensor data of Ss, the following authentication steps should be performed among $\mathrm{Um}, \mathrm{GN}$ and Ss, and a session key is shared among these three parties at the end of the authentication phase.

Step 1. Um inputs IDm and PWm on the mobile device, and imprints the biometric information $\mathrm{Bio}_{\mathrm{m}}^{\prime}$ on the mobile device with fuzzy extractor. Then the mobile device calculates.

$\sigma_{m^{\prime}}=\operatorname{Rep}\left(\mathrm{Bio}_{\mathrm{m}}^{\prime}, \tau_{m}\right)$

$\mathrm{RPW}^{\prime} \mathrm{m}=(\mathrm{PWm} \oplus \mathrm{rm})$

$\mathrm{A}^{\prime} \mathrm{m}=\mathrm{Bm} \bigoplus\left(\mathrm{RPW} W^{\prime} \mathrm{m} \bigoplus \sigma_{m^{\prime}}\right)$

checks A ' $\mathrm{m}$ ?= Am. Imbalance implies in any event one of three variables is invalid, and the login demand is rejected by the cell phone. Or disaster will be imminent, the cell phone plays out the following stage. The cell phone delivers an arbitrary numbers bm $\epsilon \mathrm{Z}_{\mathrm{n}}^{*}$ and calculates.

MM1 = bm*PP,

$\mathrm{MM} 2=\mathrm{bm}^{*} \mathrm{XX}$

$\mathrm{MM} 3=\mathrm{IDm} \oplus \mathrm{MM} 2$

$\mathrm{MM} 4=\mathrm{IDs} \oplus \mathrm{MM} 2$

$\operatorname{MM5}=\mathrm{H} 1\left(\mathrm{~A}^{\prime} \mathrm{m}\right)$
Then the mobile device(User) submits the login request \{ MM1,MM3, MM4,MM5 \} to GN.

Step 2. When receiving the request, GN first retrieves IDm, IDs and then computes A"m and then it calculates the hash value of $\mathrm{A}^{\prime \prime} \mathrm{mth}$ at is MM'5.

MM'2 $=\mathrm{xx}^{*} \mathrm{MM} 1$

ID'm =MM3 $\oplus$ MM'2

ID's =MM4 $\oplus$ MM'2

$\mathrm{A}^{\prime \prime} \mathrm{m}=\left(\mathrm{ID} ' \mathrm{~m} \oplus \mathrm{X}_{\mathrm{GN}}\right)$

MM'5=H1 (A"m)

GN server checks MM'5=MM5 If yes then the GN Server get a assurance that the user is genuine then GN server calculates the shared secret key $\mathrm{X}^{\prime}$ IDs -GN between sensor node(Ss) (given by user) and itself then it calculates MM6, MM7 for performing authentication.

$\mathrm{X}_{\text {IDs -GN }}=\left(\mathrm{ID}\right.$ 's $\left.\oplus \mathrm{X}_{\mathrm{GN}}\right)$

MM6 $=\mathrm{X}_{\text {IDs -GN }} \oplus \mathrm{ID}{ }^{\prime} \mathrm{m}$

$\mathrm{MM} 7=\mathrm{H} 1\left(\mathrm{X}_{\mathrm{IDs}-\mathrm{GN}}^{\prime}\right)$

At last, GWN Server sends \{ MM6,MM7 \} to sensor Node.

Step 3. After receiving the message from GN Server, Sensor node(Ss) first computes.

$\mathrm{MM}^{\prime} 7=\mathrm{H} 1\left(\mathrm{X}_{\mathrm{IDs}-\mathrm{GN}}\right)$

Sensor node checks MM'7=MM7, if yes then sensor node(Ss) gets a assurance that the request came from correct server, then sensor node(Ss) calculates.

ID"m = MM6 $\bigoplus X_{\text {IDs -GN }}$

MM8 =H1 $\left(X_{\text {IDs -GN }} \bigoplus\right.$ ID"m)

The Sensor node also gets the information about the user who wants to communicate, after that it sends responses $\{$ MM8\} to GN Server.

Step 4.After receiving the response from the sensor node(Ss) the GN Server retrieves computes.

MM'8 = H1 ( $\left.\mathrm{X}_{\text {IDs -GN }} \oplus \mathrm{ID}^{\prime} \mathrm{m}\right)$

if (MM'8 = MM8), GN Server gets an assurance that sensor node(Ss) is genuine and generates the session key to be given to both mobile device(user) and sensor node(Ss):

$\mathrm{SKS}=\mathrm{ID}$ 'm $\odot$ IDs $\odot \mathrm{X}_{\mathrm{GN}} \odot \mathrm{A}^{\prime \prime} \mathrm{m} \odot \mathrm{xx}$

$\mathrm{MM} 9=\mathrm{X}_{\text {IDs-GN }}^{\prime} \oplus \mathrm{SKS}$

MM10 $=$ MM'2 $\oplus$ SKS

MMM10 = H1 (A"m $\oplus$ ID'm $)$

Then GN Server sends MM9 to sensor node(Ss) and (MM10,MMM10) to mobile device(user). Otherwise, server cuts off the communication. 
Step 5. After receiving the message from GN Server, mobile device(User) computes MMM'10.

\section{MMM'10=H1(A'm $\oplus$ IDm)}

If MMM'10 = MMM10 then mobile device(user) gets a assurance that he is communicating with correct GN server then mobile device computes secret key(SKm).

\section{$\mathrm{SKm}=\mathrm{MM} 10 \oplus \mathrm{MM} 2$}

\section{MM11= H1(MM10)}

Then the mobile device(User) submits MM11 to GN Server.

Step 6.After receiving the message from GN Server, Sensor node(Ss) retrieves the secret key.

SKs $=$ MM9 $\oplus \mathrm{X}_{\mathrm{IDs}-\mathrm{GN}}$

MM12= H1(MM9)

Then the Sensor node submits MM12 to GWN Server.

Step 7.After receiving the message from mobile device(user) MM11 and Sensor nodeMM12, GN Server, computes the following.
$\mathrm{MM}^{\prime} 11=\mathrm{H} 1\left(\mathrm{MM}^{\prime} 2 \oplus \mathrm{SKS}\right)$

$\mathrm{MM}^{\prime} 12=\mathrm{H} 1\left(\mathrm{X}_{\mathrm{IDs}-\mathrm{GN}} \oplus \mathrm{SKS}\right)$

If $M^{\prime} 11=$ M11 and $M^{\prime} 12=$ M12, GN Server gets a assurance that Mobile Device(User) and sensor node(Ss) are is genuine and received the correct secret key and hence key synchronization ends.

If mutual validation and key exchange phase completes successfully, both mobile device(user) and Sensor hub(Ss) will transfer information by using the meeting key which will be computed by the GN server and given to both mobile device(user) and the sensor hub(Ss) in secured way. First source authentication is achieved, for this a one way hash function is used. The message digest is created for date $\left(\mathrm{A}_{\mathrm{m}}\right)$ which is available at both mobile device(user) and GN server, so this message digest will act as a authenticator. In the same way sensor node(Ss) and GN server mutual authenticate each other by creating a authenticator using hash function (HA160 ) by using the common data $X_{\text {IDs -GN }}$. The details of hash function(HA-160) is given below. After all the devices get assurance about other devices the GN server generates a secret key and passes it to both sensor hub(Ss) and mobile device(user) in a secured way.

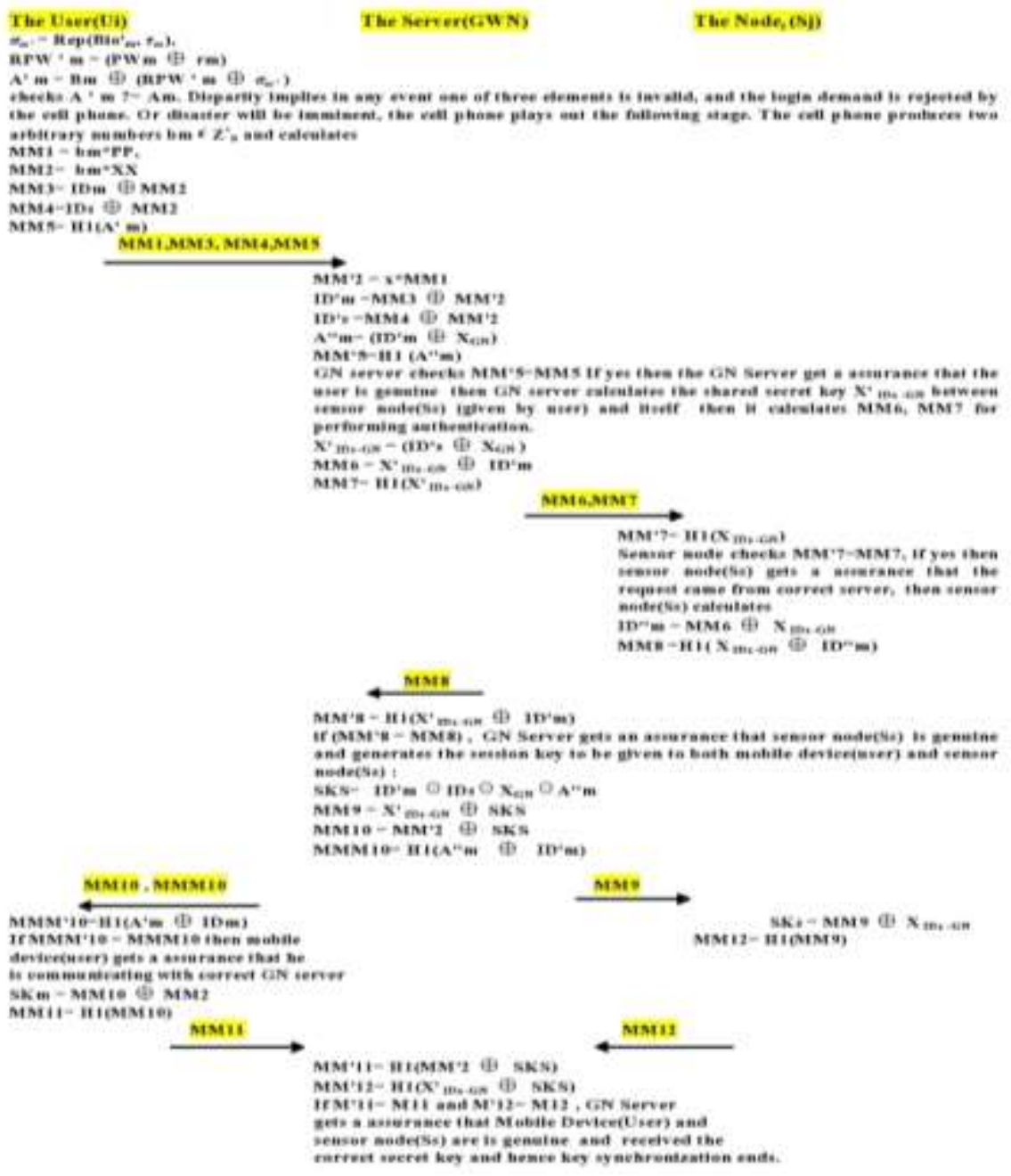

Fig. 2. Illustration of the Mutual Validation Stage and Key Synchronization Stage. 
The Secret Key between User(Ui-Mobile) and Server(GN)

The Mobile device(User-Um)

private $\mathrm{Key}=\mathrm{bm}$

$$
\begin{aligned}
& \text { Public Key }=\text { MM1 }\left(\mathrm{MM} 1=\mathrm{bm}^{* \mathrm{PP}}\right) \\
& \begin{aligned}
\text { Secret key } & =\mathrm{bm} * \mathrm{XX} \\
& =\mathrm{bm}^{*} \mathrm{xx} * \mathrm{PP}
\end{aligned}
\end{aligned}
$$

The Server(GN)

private $\mathrm{Key}=\mathrm{xx}$

Public Key $=\mathrm{XX}(\mathrm{XX}=\mathrm{XX} * \mathrm{PP})$

Secret Key $=\mathrm{xx} * \mathrm{MM} 1$

$$
=\mathrm{xx}^{*} \mathrm{bm} * \mathrm{PP}
$$

The secret key for session synchronization is showed in Fig. 2. Hash algorithm and data encryption phase is described.

\section{HASH AlgORITHM(HA-160)}

The proposed calculation is named as HA160[24][25][26]. This assessment HA-160 (Hash Algorithm) recognizes a message as responsibility with a most uncommon length of under $2^{128}$ pieces and makes a 160-piece message digest as yield. First the data message is isolated into squares of 1024 pieces. This 1024 pieces is taken as information, by then the information is diminished from 1024-digit squares to 512-cycle blocks. The Hash code creation work perceives two wellsprings of data which are 1024 pieces square of the message and the instate MD support (getting variable 160-bits). The cycle includes the going with advances.

\section{A. Attach Padding Pieces}

The message is padded so its length is reliable to 896 modulo 1024 (length $=896 \mathrm{mod}$ 1024). Padding is continually done, whether or not the message is of needed length. Subsequently, the amount of padding pieces is in the extent of 1-1024. The padding contains alone 1 followed by the significant number of 0 's for example $10 \ldots . .0$.

\section{B. Attach Length}

A square of 128 pieces which contains the length of the message (going before cushioning) is joined to the message. This square is treated as an unsigned 128-digit number (most basic byte first).

\section{Initialize MD Pad}

A 160-piece cushion is utilized to hold impermanent and eventual outcome of the hash work. The assistance can be tended to as five 32-digit registers (SS0, SS1, SS2, SS3 and SS4).These registers are instated to the going with 32-bit numbers (Hexadecimal qualities):

$$
\begin{aligned}
& \text { SS0 }=67452301 \\
& \text { SS1 }=\text { efcdab } 89 \\
& \text { SS2 }=98 \text { ba de fe } \\
& \text { SS3 }=10325476
\end{aligned}
$$

$$
\mathrm{SS} 4=\mathrm{c} 3 \mathrm{~d} 2 \text { e } 1 \text { f } 0
$$

These attributes are same as the secret vector evaluations of SHA-1 which are normalized by Federal Information Processing Standards Publications (FIPS PUBS). These qualities are dealt with in big endian plan, which is the standard byte of a word in the low-address byte position.

\section{Processes Message in 1024-Bitblocks}

The message digest age strategy contains five sub capacity. This part is named (HA-160) in Fig. 1 and its reasoning is showed up in Fig. 2. Fig. 1 depicts the general getting ready of message to make a information survey. The consequence of the underlying two phases (after add padding pieces and append size) yields a information that is a number various of 1024-piece long. The all-inclusive information is addressed as the gathering of 1024-piece blocks $\mathrm{XX}_{0}$, $\mathrm{XX}_{1}, \mathrm{XX}_{2} \mathrm{XX}_{\mathrm{L}-1}$, so the total size of the all-inclusive information is $\mathrm{L} \times 1024$ pieces $(\mathrm{L}=$ the amount of 1024 bit hinders), that is the outcome of various of 32-bit blocks. Here $\mathrm{K}$ addresses the real length of the message in pieces, "IV'" is the fundamental vector which is used to present the five 32cycle registers (SS0, SS1, SS2, SS3 and SS4). $\mathrm{VC}_{\mathrm{i}}, \mathrm{VC}_{\mathrm{ii}}, \mathrm{VC}_{\mathrm{q}}$ and $\mathrm{VC}_{\mathrm{L}-1}$ address instate $\mathrm{MD}$ (carry vector) which holds widely appealing and inevitable result of the Hash work, separately. Each round takes two data sources one 1024-cycle block $(\mathrm{XXq})$ of the message and a 160 piece pass on vector (VCq). Close to the completion of the $\mathrm{L}^{\mathrm{th}}$ stage produces 160 piece message digest. From the start the given message is disengaged into 1024-digit blocks, and each square is passed to Hash Code making capacity (HA) as a commitment close by the 160-piece vector(as shown in Fig. 3 and Fig. 4).

The Hash work $\left(\mathrm{H}_{\mathrm{HA}}-160\right)$ rationale is:

Remouldingtask $\left(\mathrm{R}_{\mathrm{fn}}\right)$ converts the given 1024-bit block into adjusted 512-bit block.

Progression $\operatorname{task}\left(\mathrm{P}_{\mathrm{fn}}\right)$ converts the given 160-piece initial vector into extended 512-bit vector.

32-digit XOR operation $\left(\mathrm{XOR}_{\mathrm{op}}\right)$ performs $\mathrm{XOR}$ procedure on each 32-pieces of altered 512-bit block and extended 512bit vector.

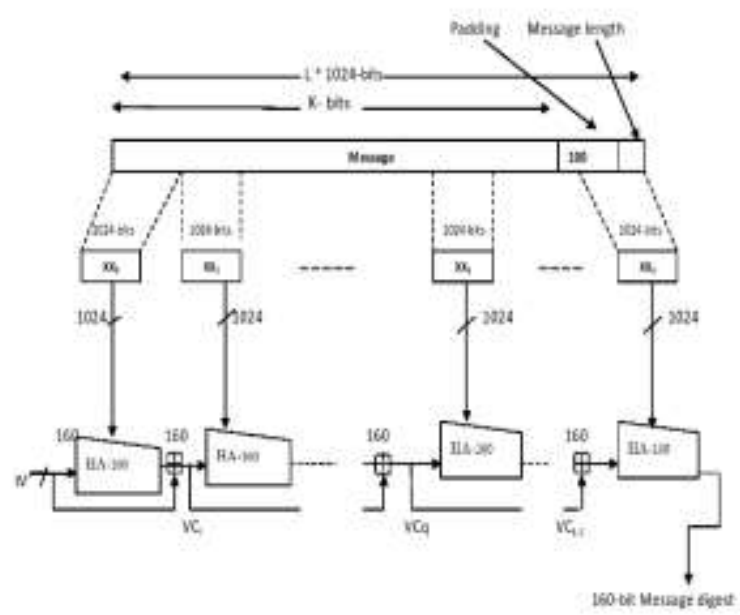

Fig. 3. Message Digest Creation using HA-160. 


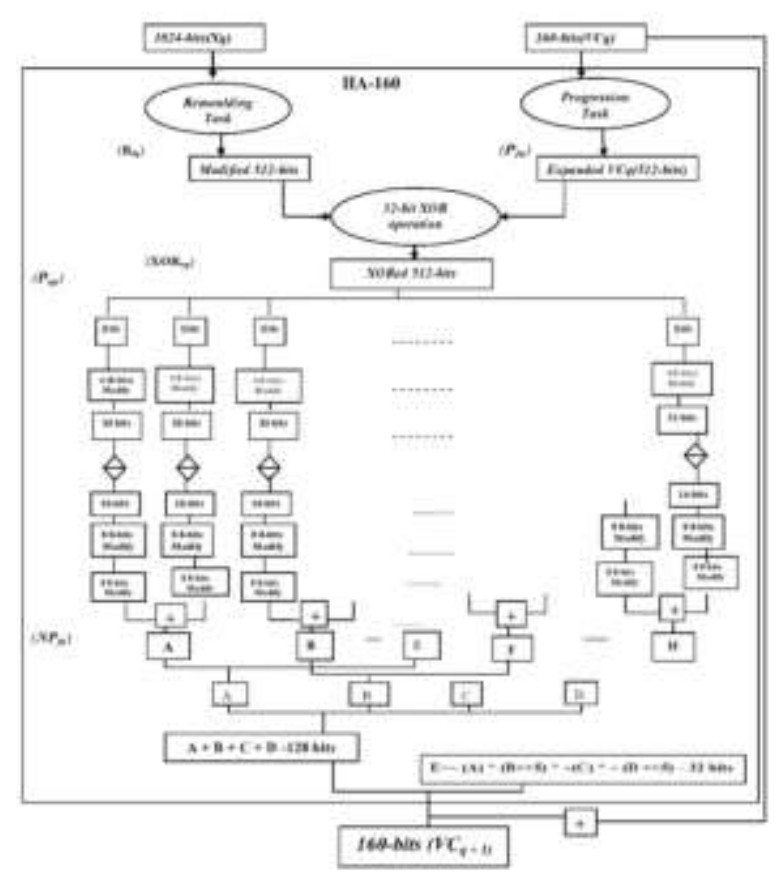

Fig. 4. The Rationale of Hash Work (Compression Work).

Partition and Modification operation $\left(\mathrm{P}_{\mathrm{op}}\right)$ disconnects the 512-cycle block into 16 sub-square of 32-bits each and afterward each 32 digit block is isolated into four 8-bit block, after that it changes every eight bit block. This interaction is rehashed for every one of the 32 bit block.

New Point(on elliptic curve) estimation work $\left(\mathrm{NP}_{\mathrm{fn}}\right)$ calculates new point on elliptic curve using 4 eight-cycle block(from each 32-bit block).

Where,

- $\mathrm{XXq}=$ the qth 1024-cycle square of the information.

- $\mathrm{VCq}=$ mooring variable arranged with the qth square of the information.

- $\mathrm{Rfn}=$ Remoulding task.

- $\mathrm{P}=(\mathrm{xxx} 1, \mathrm{yyy} 1)$ and $\mathrm{Q}=(\mathrm{xxx} 2, \mathrm{yyy} 2)$.

- $\mathrm{Pfn}=$ Progression task.

- XORop=XOR(Exclusive-OR) development performed on each 32-cycle square of the adjusted 512-digit block (XXq) and relating 32-pieces of the comprehensive 512-bit block (VCq).

- Pop = 512-digit square can be withdrawn into $16 \mathrm{sub}$ squares of 32-bits each and subsequently each 32 cycle block is confined into four 8-bit block, after that it changes each eight bit block.

- $\mathrm{NPfn}=$ initial 32-cycle square further parceled into 2 sub parts of 16-piece each.

1 stsub part $=16$ bit, apportioned into two sub part (8bits, 8 -bits $)=(\operatorname{xxx} 1$, yyy 1$)$. 2nd sub part $=16$ bit, divided into two sub part (8-bits, 8 bits $)=(\mathrm{xxx} 2, \mathrm{yyy} 2)$.

The characteristics (xxx1, yyy1, $\mathrm{xxx} 2$, and yyy2 as showed in Fig. 5) are changed over into entire numbers followed by processing another point on elliptic curve. The above interaction is revamped for staying fifteen 32-cycle block and the outcome is 16 sub square every one of 16 pieces. Presently every one of this 8 sub square every one of 16 digit is changed, the initial 8-cycle of first square is XOR with a 8-bit sub square which are each of the zeros, the outcome is put away in initial 8-bit sub square. The subsequent 8-digit sub square of first square is added with initial 8-bit sub square and result is put away in second 8-cycle sub square and the interaction is rehashed for every one of the 8-piece sub square. The outcome is arranged 256 cycle block. Presently neighboring sub square every 16 pieces are added which brings about a sub square of 32 digit and we get eight 32 piece sub square. At that point this eight 32-digit sub square are converted to four 32-bit sub block by added first and fifth, second and sixth, third and seventh and fourth and eight 32digit sub square are added which brings about four 32-cycle sub square. By playing out some numerical procedure on over four processed 32 bit sub square and afterward XOR them brings about the fifth 32 cycle sub square. This five 32 piece sub square when concatenated adds up to 160 piece and when summed with introduced MD cradle shapes the hash code. This 160 piece hash code will be the contribution to next 1024 digit of message i.e it will go about as introduce MD cradle for next 1024 cycle of the info message. The last 160 piece code produced from last 1024 cycle of message will be the last message digest which will go about as confirmation code.

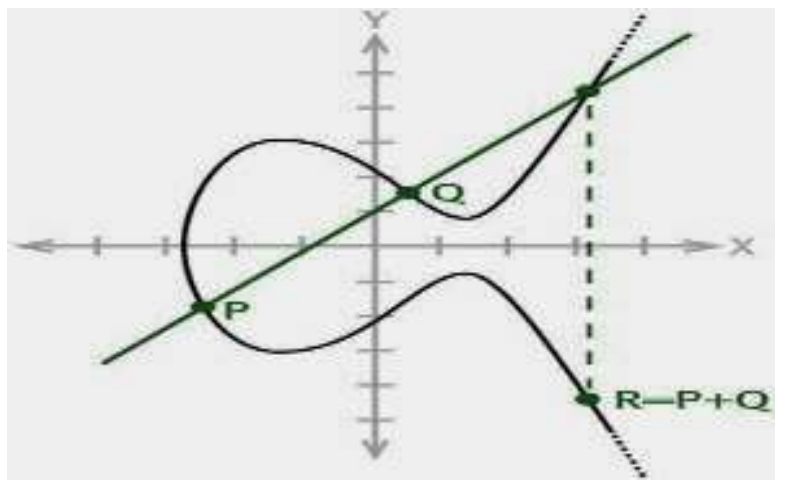

Fig. 5. Elliptic Curve Way to Represent Two Points.

1) Remouldingtask $\left(R_{f n}\right)$ :Each 1024-cycle square of data is isolated into 128 sub-blocks including 8-bits of each subblock. One brief show of size 8 (Tempx8) is taken and instated with zeroes. The adjustment work includes two sub task as shown as follows.

Sub-work 1. Initially, the first 8-bits of 1024 block is XORed with Tempx8 variable i.e eight bit starting from least significant bit in Tempx8 is XORed with first 8-bits of 1024 block. the result is stored in first 8-bit of 1024 block(Modified Message) and Tempx 8 is incremented by one. This extended Tempx8 is XOR with the going with 8-pieces of the message to make the going with 8-pieces of changed message as tended to in Fig. 6. 


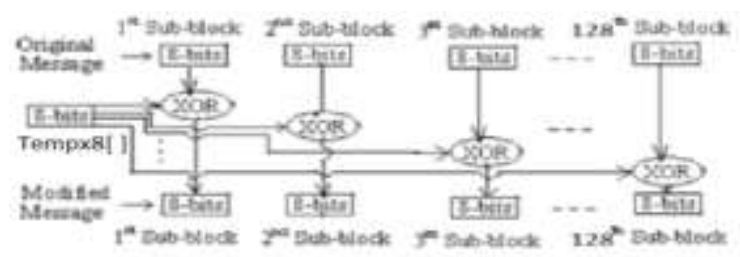

Fig. 6. Function of Sub-Work1.

Sub-work 2. The above result is again detached into sub square of 8 pieces and initial eight-cycle sub square and focus eight-bit sub square after bitwise complimenting( ) are XOR and the result is again bitwise complimented $(\sim)$ and set aside in an alternate array(W2). The above cycle is reiterated for second 8-bit sub square and focus notwithstanding one 8-digit sub square and result is taken care of in second piece of disconnected array(W2). This is repeated for all extra 8-digit sub square. The result is 1024 cycle block is diminished to 512 digit block. The changed message as depicted under.

intMod_Inp(vector $<$ int $>\mathrm{mm}$, intnn)

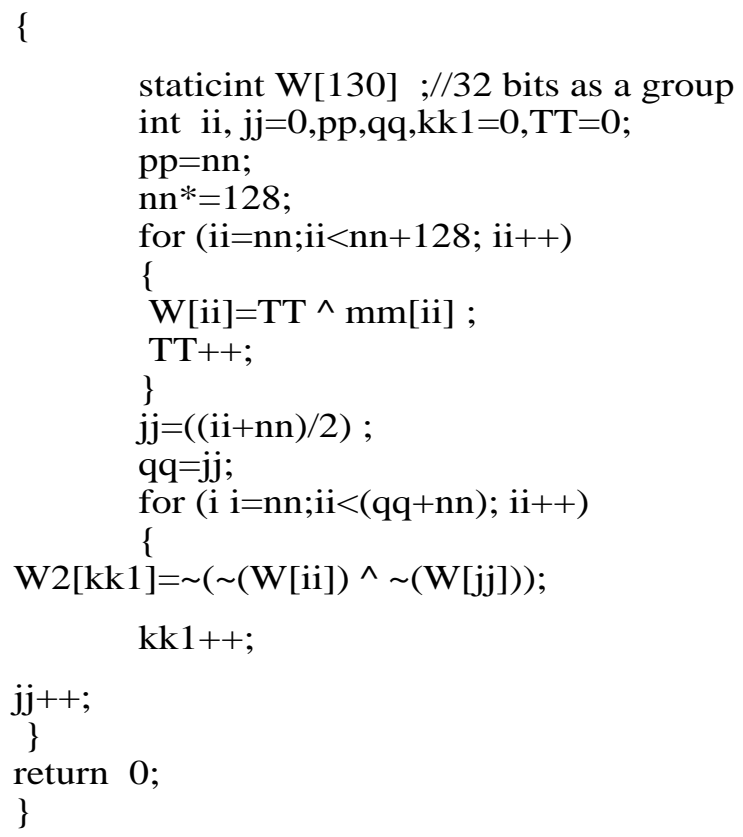

2) ProgressionTask $\left(P_{f n}\right)$ :The 160-piece Initial Vector (IV) is one of the commitments to the message digest creation work (HA-160). It will in general be stretched out to 512-bits by interfacing all hidden vector regards in indirect manner, which is called Concatenated Vector (VC). The association cycle is showed in Fig. 7.

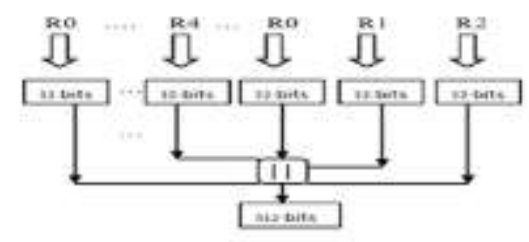

Fig. 7. Formation of 160-Bit Block (IV') to 512-Bit Block (CV).
3) 32-bit $X O R$ operation $\left(X O R_{\text {op }}\right): X O R$ movement is done on starting 32-piece sub-block of modified 512-cycle square and expanded 512-piece square. For this initial four 8-digit block are extended and summed with the goal that it becomes 32 piece square. At that point this 32-piece square is XOR with initial 32-bit of extended or instate MD cradle of 512piece square. This interaction is rehashed for rest of the pieces of adjusted 512-bit and extended 512-digit block. The outcome is 512-cycle block as demonstrated underneath.

$$
\text { for }(\mathrm{tt} 1=0 ; \mathrm{tt} 1<16 ; \mathrm{tt} 1++)
$$

$\mathrm{WW} 3[\mathrm{t} 1]=(\mathrm{WW} 2[4 * \mathrm{t} \mathrm{t} 1]<<24)+(\mathrm{WW} 2[4 * \mathrm{tt} 1$ $+1]<<16)+(\mathrm{WW} 2[4 * \mathrm{tt} 1+2]<<8)+(\mathrm{WW} 2[4 *$ tt $1+3])$

if $(\mathrm{tt} 1==0)$

$\mathrm{WW} 3[\mathrm{tt} 1]=((\mathrm{WW} 3[\mathrm{tt} 1]) \wedge(\mathrm{EE}))$;

if $(\mathrm{tt} 1==1)$

$\mathrm{WW} 3[\mathrm{t} 1]=\left((\mathrm{WW} 3[\mathrm{t} 1])^{\wedge}(\mathrm{DD})\right)$;

$\operatorname{if}(\mathrm{tt} 1==2)$

$\mathrm{WW} 3[\mathrm{t} 1]=\left((\mathrm{WW} 3[\mathrm{tt} 1])^{\wedge}(\mathrm{CC})\right)$;

if $(\mathrm{tt} 1==3)$

$\mathrm{WW} 3[\mathrm{tt} 1]=\left((\mathrm{WW} 3[\mathrm{tt} 1])^{\wedge}(\mathrm{BB})\right)$;

if $(\mathrm{tt} 1==4)$

$\mathrm{WW} 3[\mathrm{tt} 1]=\left((\mathrm{WW} 3[\mathrm{tt} 1])^{\wedge}(\mathrm{AA})\right)$;

if $(\mathrm{tt} 1==5)$

$\mathrm{WW} 3[\mathrm{tt} 1]=(\mathrm{WW} 3[\mathrm{tt} 1]) \wedge(\mathrm{EE})$;

if $(\mathrm{tt} 1==6)$

$\mathrm{WW} 3[\mathrm{tt} 1]=((\mathrm{WW} 3[\mathrm{tt} 1]) \wedge(\mathrm{DD}))$;

if $(\mathrm{tt} 1==7)$

$\mathrm{WW} 3[\mathrm{tt} 1]=(\mathrm{WW} 3[\mathrm{tt} 1])^{\wedge}(\mathrm{CC})$;

if $(\mathrm{tt} 1==8)$

$\mathrm{WW} 3[\mathrm{tt} 1]=(\mathrm{WW} 3[\mathrm{tt} 1])^{\wedge}(\mathrm{BB})$;

if $(\mathrm{tt} 1==9)$

$\mathrm{WW} 3[\mathrm{tt} 1]=(\mathrm{WW} 3[\mathrm{tt} 1])^{\wedge}(\mathrm{AA})$;

if $(\mathrm{tt} 1==10)$

$\mathrm{WW} 3[\mathrm{tt} 1]=(\mathrm{WW} 3[\mathrm{tt} 1])^{\wedge}(\mathrm{EE})$;

if $(\mathrm{tt} 1==11)$

$\mathrm{WW} 3[\mathrm{tt} 1]=(\mathrm{WW} 3[\mathrm{tt} 1]) \wedge(\mathrm{DD})$;

if $(\mathrm{tt} 1==12)$

$\mathrm{WW} 3[\mathrm{tt} 1]=(\mathrm{WW} 3[\mathrm{tt} 1])^{\wedge}(\mathrm{CC})$;

if $(\mathrm{tt} 1==13)$

$\mathrm{WW} 3[\mathrm{tt} 1]=(\mathrm{WW} 3[\mathrm{tt} 1])^{\wedge}(\mathrm{BB})$;

if $(\mathrm{tt} 1==14)$ 
$\mathrm{WW} 3[\mathrm{tt} 1]=(\mathrm{WW} 3[\mathrm{tt} 1])^{\wedge}(\mathrm{AA}) ;$

if $(\mathrm{tt} 1==15)$

$\mathrm{WW} 3[\mathrm{tt} 1]=(\mathrm{WW} 3[\mathrm{tt} 1])^{\wedge}(\mathrm{EE})$;

$$
\text { \} }
$$$$
\text { \} }
$$

4) Partition and Modification operation $\left(P_{o p}\right)$ :Each 32-digit square of the above outcome is additionally allocated 2 sub-squares of 16-bits each. By then every 16-bit block is segregated into two 8-cycle sub square, all of these eight pieces are changed that is First the four pieces are taken from the 8-digit regard starting from least basic position then it is XOR with a variable $1(\mathrm{~mm})$ whose value is zero, the result is kept in a variable2(mm1) also the assessment of variable $1(\mathrm{~mm})$ is invigorated for instance it is given the assessment of variable2( $\mathrm{mm} 1)$. Ensuing to completing the above advance again from 8-digit value(input), four pieces starting from most critical position is taken care of in variable $3(\mathrm{~mm} 2)$ and variable 3 is changed by playing out a XOR with variable1(mm). The data is reproduced for example 8-cycle regard is recomputed by taking care of the assessment of variable $3(\mathrm{~mm} 2)$ (four bits starting from least basic situation) in four pieces starting from most huge position and next four pieces of early on eight bit(input) communicating from least critical position gets the pieces from variable2(km1) (four pieces starting from least huge position) so this way the 8-digit of data is changed. A comparative procedure is embraced for remaining three 8-bit regards made from 32 cycle that is

$\mathrm{BB} 1=(\mathrm{WW} 3[\mathrm{t}]>8) \& 0 \mathrm{xff} ; \rightarrow 2^{\text {nd }} 8$ - piece number made from 32 bit number

AA2 $=($ WW3 $[\mathrm{t}]>>16) \quad \&$ oxff $\rightarrow 3^{\text {rd }} 8$ cycle number made from 32 bit number

$\mathrm{BB} 2=(\mathrm{WW} 3[\mathrm{t}]>24) \&$ 0xff $\rightarrow 4^{\text {th }} 8$ digit number made from 32 cycle number

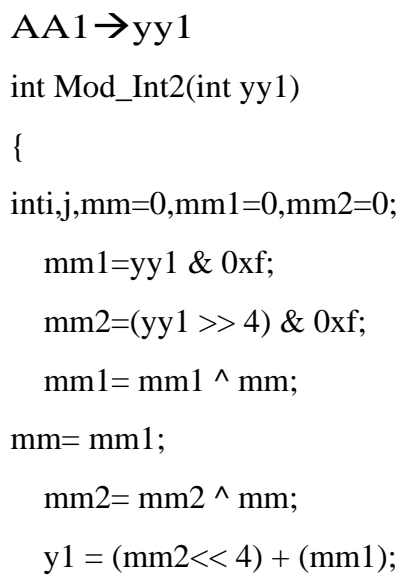

A same strategy is adopted for remaining seven 32-digit blocks.

5) New Point(on elliptic curve) estimation task $\left(N P_{f n}\right)$ :Each 32-digit block is separated into two sub squares of same length. These sub squares go probably as two motivations behind an elliptic curve which are used in new point appraisal in elliptic curve as exhibited in Fig. 5. First by using two point slope $(\lambda)$ of line is resolved the this slant value(M) is used in the calculation of new $\mathrm{X}$ and $\mathrm{Y}$ center point on elliptic curve. The resultant new point is 16 cycle regard i.e $\mathrm{X}$ center is 8 pieces and $\mathrm{Y}$ center point is 8 pieces so both taken together is 16 digit sub square. An amount of sixteen 16-cycle sub square is created after new point computation. As of now this new point are changed by performing XOR on new point. Each 8-digit sub square is XOR with its adjoining 8-cycle sub square beside beginning 8-bit sub square which is XOR with a 8 bit sub square, everything being zero. After above action connecting sub squares are incorporated an especially that it becomes 32 cycle sub square. Repeating this, achieves eight 32-digit sub square.

Incline of line in elliptic curve (for real) is determined as:

$\operatorname{slope}(\lambda)=\frac{(\text { yyy } 2-\text { yyy } 1)}{(x x \times 2-x x x 1)}$

where (xxx1,yyy1) and (xxx2,yyy2) are points on elliptic curve. New point on elliptic curve for reals are calculated using the following formula

$\mathrm{xx}_{3}($ new point $)=(\operatorname{slope}(\lambda))^{2}-\mathrm{xxx}_{1}-\mathrm{xxx}_{2}$

and

$\mathrm{yy}_{3}($ new point $)=\operatorname{slope}(\lambda) *\left(\mathrm{xxx}_{1}-\mathrm{xxx}_{2}\right)-\mathrm{yyy}_{1}$

the above calculation is for the case when $\operatorname{xxx} 1 !=$ (not equal to) $\mathrm{xxx} 2$ (this is assumed that $\mathrm{xxx} 1$ is not equal to $\mathrm{xxx} 2$ ). The logic of new point estimation is demonstrated below.

$$
\begin{aligned}
& \text { for }(\mathrm{tt}=0 ; \mathrm{tt}<16 ; \mathrm{tt}++) \\
& \{ \\
& \mathrm{AA} 1=\mathrm{WW} 3[\mathrm{tt}] \& 0 \mathrm{xff} \text {; } \\
& \text { AA1=(Mod_Int2(AA1) }) \text {; } \\
& \mathrm{BB} 1=(\mathrm{WW} 3[\mathrm{tt}]>>8) \& 0 x f f \text {; } \\
& \text { BB 1 = (Mod_Int2(BB1)); } \\
& \mathrm{AA} 2=(\mathrm{WW} 3[\mathrm{tt}]>>16) \& 0 \mathrm{xff} \text {; } \\
& \text { AA2 }=(\text { Mod_Int2(AA2) }) \text {; } \\
& \mathrm{BB} 2=(\mathrm{WW} 3[\mathrm{tt}]>>24) \& 0 x f f ; \\
& \text { BB2 }=(\text { Mod_Int2(BB2) }) \text {; } \\
& \mathrm{MM}=(\mathrm{BB} 2-\mathrm{BB} 1) /(\mathrm{AA} 2-\mathrm{AA} 1) \text {; } \\
& \text { AA3[ii] }=(M M * M M)-A A 1-A A 2 \text {; } \\
& \text { AA3[ii }]=A A 3[i i]^{\wedge} \text { TT } 1 \text {; } \\
& \text { BB3[ii] }=\mathrm{MM}^{*} \text { (AA1-AA2)-BB1; } \\
& \mathrm{BB} 3[\mathrm{ii}]=\mathrm{BB} 3[\mathrm{ii}] \wedge \text { AA3[ii]; } \\
& \text { TT1=BB3[ii]; }
\end{aligned}
$$


ii++;

\section{\}}

The above process results in A3 and B3 array. Now each of this array element is altered using the strategy

for $(\mathrm{ii}=0 ; \mathrm{ii}<16 ; \mathrm{ii}=\mathrm{ii}+2)$

\{

if(ii<2)

\section{\{}

$\mathrm{AA} 3[\mathrm{ii}]=(\mathrm{AA} 3[\mathrm{ii}]<24)+(\mathrm{BB} 3[\mathrm{ii}]<<16)+(\mathrm{AA} 3[\mathrm{ii}+1]$ $<<8)+($ BB3 $[\mathrm{ii}+1])$

$\mathrm{BB} 3[\mathrm{ii}]=(\mathrm{BB} 3[\mathrm{ii}]<<24)+(\mathrm{AA} 3[\mathrm{ii}]<<16)+(\mathrm{BB} 3[\mathrm{ii}+1]$ $<<)+($ AA3 $[\mathrm{ii}+1])$

$\mathrm{AA} 3[\mathrm{ii}+1]=(\mathrm{AA} 3[\mathrm{ii}+1]<<24)+(\mathrm{BB} 3[\mathrm{ii}+1]<<16)+$ $(\mathrm{AA} 3[\mathrm{ii}]<<8)+(\mathrm{BB} 3[\mathrm{ii}])$;

$\mathrm{BB} 3[\mathrm{ii}+1]=(\mathrm{BB} 3[\mathrm{ii}+1]<<24)+(\mathrm{AA} 3[\mathrm{ii}+1]<<16)+$ $($ BB3 $[\mathrm{ii}]<<8)+($ AA3 $[\mathrm{ii}])$

\}

if(ii $>=2 \& \&$ ii $<4)$

\{

$\mathrm{AA} 3[\mathrm{ii}]=(\mathrm{AA} 3[\mathrm{ii}]<24)+(\mathrm{BB} 3[\mathrm{ii}]<<16)+(\mathrm{AA} 3[\mathrm{ii}+1]$ $<<8)+(\mathrm{BB} 3[\mathrm{ii}+1])$

$\mathrm{BB} 3[\mathrm{ii}]=(\mathrm{BB} 3[\mathrm{ii}]<<24)+(\mathrm{AA} 3[\mathrm{ii}]<<16)+(\mathrm{BB} 3[\mathrm{ii}+1]$ $<<)+(\mathrm{AA} 3[\mathrm{ii}+1])$

$\mathrm{AA} 3[\mathrm{ii}+1]=(\mathrm{AA} 3[\mathrm{ii}+1]<24)+(\mathrm{BB} 3[\mathrm{ii}+1]<<16)+$ $(\mathrm{AA} 3[\mathrm{ii}]<<8)+($ BB3 $[\mathrm{ii}])$

$\mathrm{BB} 3[\mathrm{ii}+1]=(\mathrm{BB} 3[\mathrm{ii}+1]<<24)+(\mathrm{AA} 3[\mathrm{ii}+1]<<16)+$ $($ BB3 $[\mathrm{ii}]<<8)+($ AA3 $[\mathrm{ii}])$

\section{\}}

if $(\mathrm{ii}>=4 \& \& \mathrm{ii}<6)$

\{

$\mathrm{AA} 3[\mathrm{ii}]=(\mathrm{AA} 3[\mathrm{ii}]<<24)+(\mathrm{BB} 3[\mathrm{ii}]<<16)+(\mathrm{AA} 3[\mathrm{ii}+1]$ $<<8)+(\mathrm{BB} 3[\mathrm{ii}+1])$

$\mathrm{BB} 3[\mathrm{ii}]=(\mathrm{BB} 3[\mathrm{ii}]<<24)+(\mathrm{AA} 3[\mathrm{ii}]<<16)+(\mathrm{BB} 3[\mathrm{ii}+1]$ $<<)+($ AA $3[\mathrm{ii}+1])$

$\mathrm{AA} 3[\mathrm{ii}+1]=(\mathrm{AA} 3[\mathrm{ii}+1]<24)+(\mathrm{BB} 3[\mathrm{ii}+1]<16)+$ $(\mathrm{AA} 3[\mathrm{ii}]<<8)+($ BB3 $[\mathrm{ii}])$

$\mathrm{BB} 3[\mathrm{ii}+1]=(\mathrm{BB} 3[\mathrm{ii}+1]<<24)+(\mathrm{AA} 3[\mathrm{ii}+1]<<16)+$ $(\mathrm{BB} 3[\mathrm{ii}]<<8)+(\mathrm{AA} 3[\mathrm{ii}])$

\section{\}}

if(ii $>=6 \& \&$ ii $<8)$

\{

$\mathrm{AA} 3[\mathrm{ii}]=(\mathrm{AA} 3[\mathrm{ii}]<<24)+(\mathrm{BB} 3[\mathrm{ii}]<<16)+(\mathrm{AA} 3[\mathrm{ii}+1]$ $<<)+(\mathrm{BB} 3[\mathrm{ii}+1])$

$\mathrm{BB} 3[\mathrm{ii}]=(\mathrm{BB} 3[\mathrm{ii}]<<24)+(\mathrm{AA} 3[\mathrm{ii}]<<16)+(\mathrm{BB} 3[\mathrm{ii}+1]$ $<<8)+(\mathrm{AA} 3[\mathrm{ii}+1])$

$\mathrm{AA} 3[\mathrm{ii}+1]=(\mathrm{AA} 3[\mathrm{ii}+1] \ll 24)+(\mathrm{BB} 3[\mathrm{ii}+1] \ll 16)+$ $(\mathrm{AA} 3[\mathrm{ii}]<<8)+(\mathrm{BB} 3[\mathrm{ii}])$

$\mathrm{BB} 3[\mathrm{ii}+1]=(\mathrm{BB} 3[\mathrm{ii}+1]<<24)+(\mathrm{AA} 3[\mathrm{ii}+1]<<16)+$ $($ BB3 $[$ ii] $<<8)+($ AA3 $[$ ii $])$

\}

if(ii $>=8 \& \&$ ii $<10)$
$\mathrm{AA} 3[\mathrm{ii}]=(\mathrm{AA} 3[\mathrm{ii}]<24)+(\mathrm{BB} 3[\mathrm{ii}]<<16)+(\mathrm{AA} 3[\mathrm{ii}+1]$ $<<8)+($ BB3 $[i i+1])$

$\mathrm{BB} 3[\mathrm{ii}]=(\mathrm{BB} 3[\mathrm{ii}]<<24)+(\mathrm{AA} 3[\mathrm{ii}]<<16)+(\mathrm{BB} 3[\mathrm{ii}+1]$ $<<8)+(\mathrm{AA} 3[\mathrm{ii}+1])$

$\mathrm{AA} 3[\mathrm{ii}+1]=(\mathrm{AA} 3[\mathrm{ii}+1]<<24)+(\mathrm{BB} 3[\mathrm{ii}+1]<<16)+$ $(\mathrm{AA} 3[\mathrm{ii}]<<8)+(\mathrm{BB} 3[\mathrm{ii}])$

$\mathrm{BB} 3[\mathrm{ii}+1]=(\mathrm{BB} 3[\mathrm{ii}+1]<<24)+(\mathrm{AA} 3[\mathrm{ii}+1]<<16)+$ $($ BB3 $[\mathrm{ii}]<<8)+($ AA3 $[\mathrm{ii}])$;

\}

if(ii $>=10 \& \&$ ii $<12)$

\{

$\mathrm{AA} 3[\mathrm{ii}]=(\mathrm{AA} 3[\mathrm{ii}]<<24)+(\mathrm{BB} 3[\mathrm{ii}]<<16)+(\mathrm{AA} 3[\mathrm{ii}+1]$ $<<)+($ BB3 $[i i+1])$;

$\mathrm{BB} 3[\mathrm{ii}]=(\mathrm{BB} 3[\mathrm{ii}]<<24)+(\mathrm{AA} 3[\mathrm{ii}]<<16)+(\mathrm{BB} 3[\mathrm{ii}+1]$ $<<)+(\mathrm{AA} 3[\mathrm{ii}+1])$

$\mathrm{AA} 3[\mathrm{ii}+1]=(\mathrm{AA} 3[\mathrm{ii}+1]<24)+(\mathrm{BB} 3[\mathrm{ii}+1]<<16)+$ $($ AA3 $[i i]<<8)+($ BB3 $[i i])$;

$\mathrm{BB} 3[\mathrm{ii}+1]=(\mathrm{BB} 3[\mathrm{ii}+1]<<24)+(\mathrm{AA} 3[\mathrm{ii}+1]<<16)+$ $($ BB3 $[$ ii $]<<8)+($ AA3 $[$ ii $])$

\}

if(ii $>=12 \& \&$ ii $<14)$

\{

$\mathrm{AA} 3[\mathrm{ii}]=(\mathrm{AA} 3[\mathrm{ii}]<<24)+(\mathrm{BB} 3[\mathrm{ii}]<<16)+(\mathrm{AA} 3[\mathrm{ii}+1]$ $<8)+($ BB3 $[\mathrm{ii}+1])$

$\mathrm{BB} 3[\mathrm{ii}]=(\mathrm{BB} 3[\mathrm{ii}]<<24)+(\mathrm{AA} 3[\mathrm{ii}]<<16)+(\mathrm{BB} 3[\mathrm{ii}+1]$ $<<)+(\mathrm{AA} 3[\mathrm{ii}+1])$

$\mathrm{AA} 3[\mathrm{ii}+1]=(\mathrm{AA} 3[\mathrm{ii}+1]<24)+(\mathrm{BB} 3[\mathrm{ii}+1]<<16)+$ $(\mathrm{AA} 3[\mathrm{ii}]<<8)+(\mathrm{BB} 3[\mathrm{ii}])$

$\mathrm{BB} 3[\mathrm{ii}+1]=(\mathrm{BB} 3[\mathrm{ii}+1]<<24)+(\mathrm{AA} 3[\mathrm{ii}+1]<<16)+$ $($ BB3 $[\mathrm{ii}]<<8)+($ AA3 $[\mathrm{ii}])$

\}

if(ii $>=14 \& \&$ ii $<16)$

\{

$\mathrm{AA} 3[\mathrm{ii}]=(\mathrm{AA} 3[\mathrm{ii}]<<24)+(\mathrm{BB} 3[\mathrm{ii}]<<16)+(\mathrm{AA} 3[\mathrm{ii}+1]$ $<8)+($ BB3 $[\mathrm{ii}+1])$

$\mathrm{BB} 3[\mathrm{ii}]=(\mathrm{BB} 3[\mathrm{ii}]<<24)+(\mathrm{AA} 3[\mathrm{ii}]<<16)+(\mathrm{BB} 3[\mathrm{ii}+1]$ $<<)+(\mathrm{AA} 3[\mathrm{ii}+1])$

$\mathrm{AA} 3[\mathrm{ii}+1]=(\mathrm{AA} 3[\mathrm{ii}+1] \ll 24)+(\mathrm{BB} 3[\mathrm{ii}+1] \ll 16)+$ $(\mathrm{AA} 3[\mathrm{ii}]<<)+(\mathrm{BB} 3[\mathrm{ii}])$

$\mathrm{BB} 3[\mathrm{ii}+1]=(\mathrm{BB} 3[\mathrm{ii}+1]<<24)+(\mathrm{AA} 3[\mathrm{ii}+1]<<16)+$ $($ BB3 $[\mathrm{ii}]<<8)+($ AA3 $[\mathrm{ii}])$

\section{\}}

\}

that is $\mathrm{AA} 3[\mathrm{i}]$ is recreated from $\mathrm{AA} 3[\mathrm{i}], \mathrm{BB} 3[\mathrm{i}], \mathrm{AA} 3[\mathrm{i}+1]$ and $\mathrm{BB} 3[\mathrm{i}+1]$ i.e. here $\mathrm{AA} 3[\mathrm{i}]$ is left shifted by 24 bits, BB3[i] is left shifted by 16 bits, AA3[i+1] is left shifted by 8 bits and $\mathrm{BB} 3[\mathrm{i}+1]$ is left shifted by 0 (zero) bits and then AA3[i], BB3[i], AA3[i+1] and BB3[i+1] are added to generate AA3[i]. Similarly BB3[i] is recreated from AA3[i], $\mathrm{BB} 3[\mathrm{i}], \mathrm{AA} 3[\mathrm{i}+1]$ and $\mathrm{BB} 3[\mathrm{i}+1]$ i.e. here BB3[i] is left shifted by 24 bits, AA3[i] is left shifted by 16 bits, BB3[i+1] is left shifted by 8 bits and AA3[i+1] is left shifted by 0 (zero) bits and then $\mathrm{AA} 3[\mathrm{i}], \mathrm{BB} 3[\mathrm{i}], \mathrm{AA} 3[\mathrm{i}+1]$ and $\mathrm{BB} 3[\mathrm{i}+1]$ are added to generate $\mathrm{BB} 3[\mathrm{i}]$. For recalculating $\mathrm{AA} 3[\mathrm{i}+1]$ again AA3[i], 
$\mathrm{BB} 3[\mathrm{i}], \mathrm{AA} 3[\mathrm{i}+1]$ and $\mathrm{BB} 3[\mathrm{i}+1]$ are used i.e. here $\mathrm{AA} 3[\mathrm{i}+1]$ is left shifted by 24 bits, BB3[i+1] is left shifted by 16 bits, AA3[i] is left shifted by 8 bits and BB3[i] is left shifted by 0 (zero) bits and then AA3[i], BB3[i], AA3[i+1] and BB3[i+1] are added to generate $\mathrm{AA} 3[\mathrm{i}+1]$. Finally for recalculating $\mathrm{BB} 3[\mathrm{i}+1]$ again $\mathrm{AA} 3[\mathrm{i}], \mathrm{BB} 3[\mathrm{i}], \mathrm{AA} 3[\mathrm{i}+1]$ and $\mathrm{BB} 3[\mathrm{i}+1]$ are used i.e. here $B B 3[i+1]$ is left shifted by 24 bits, AA3 $[i+1]$ is left shifted by 16 bits, BB3[i] is left shifted by 8 bits and AA3[i] is left shifted by 0(zero) bits and then AA3[i], BB3[i], $\mathrm{AA} 3[\mathrm{i}+1]$ and $\mathrm{BB} 3[\mathrm{i}+1]$ are added to generate $\mathrm{BB} 3[\mathrm{i}+1]$. The same process is repeated for all the value of ' $\mathrm{ii}$ '.Here ' $\mathrm{ii}$ ' is incremented by 2 when the statements inside looping structure are rotated using $\mathrm{ii}$

6) Result:The 160-piece hash code is acquired by adding four 32 bit sub square i.e. first and fifth, second and sixth, third and seventh and fourth and eight 32-bit sub square are added which brings about four 32-digit sub square. Presently this four 32-cycle block got in above interaction are numerically controlled which brings about last 32-digit sub square as demonstrated beneath. The message digest (160 pieces) is gotten by connecting the five 32-cycle block got in above interaction.

$$
\begin{aligned}
& \mathrm{EE}=\sim(\mathrm{AA})^{\wedge}(\mathrm{BB}>>5)^{\wedge} \sim(\mathrm{CC})^{\wedge} \sim(\mathrm{DD}<<5) \\
& \text { 160-bit Hash Code }=\mathrm{AA}\|\mathrm{BB}\| \mathrm{CC}\|\mathrm{DD}\| \mathrm{EE}
\end{aligned}
$$

All hexadecimal numbers are copied into pass on vector (or instate MD cushion), which is the message blueprint of a given message(if the message size is 1024-piece square) in any case the above cycle is emphasized until the last 1024digit square of the message.

\section{ENCRYPTION ALGORITHM}

Step 1: In trigonometry operation, for encryption we use a trigonometric formula made up of $\operatorname{Cos}$ function i.e. Cos $(x * p i / 180.0)=y$ then $\operatorname{Sin}$ function i.e. $\operatorname{Sin}(y * p i / 180.0)$ $=y 1$, here we require the value of $\pi$ for $\operatorname{Cos}$ and $\operatorname{Sin}$ function. At the time of encryption and decryption this value of pi will be needed. The actual value of $=3.141592$, but in this case the Sensor Nodeand mobile device(user)will take the value of $\pi$ be current key generated from the secret key for session $\mathrm{SK}_{\mathrm{m}}$ or $\mathrm{SK}_{\mathrm{s}}$. For example, let the value of key be 77 .

Here is the method how the key for encryption or decryption is generated from secret key for session(SKi).

1) First the secret key is stored in a variable and then this variable is rotated ten times, for each rotation it checks whether the rotation is even or odd. If even, the variable is right shifted by one and the shifted value is stored in a new $\operatorname{array}(\mathrm{xx})$, also the variable gets the current value of array $\mathrm{x}$ and the array is incremented. If odd, the variable is left shifted by one and the shifted value is stored in a new array(xx), also the variable gets the current value of array $\mathrm{x}$ and array is incremented. This process is repeated for a array of size ten, so at the end of first step we get ten different values generated from secret key. The process is shown below:

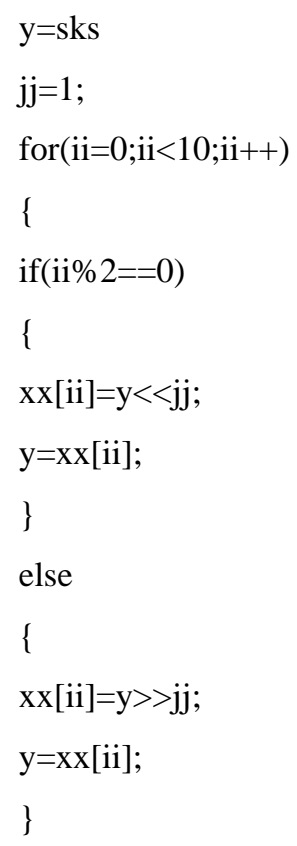

2) Now the new array is analyzed that is from secret key ten different keys are generated in stage1, then this ten keys are XOR and the result of XOR operation is kept in a variable(t). After computing the value of $t$, the new array is modified that is array $x x$ first position will now hold the value of array xx second position and array xx second position will now hold the value of array $x x$ third position and the process continues till ninth position tenth array $\mathrm{xx}$ position will hold the value of variable t. After completion of above step one position of array $\mathrm{xx}$ (tenth position) is altered, the same process is repeated for all the position(ninth to one position) of array xx. So after stage 2 the ten different key generated in step one will be holding all new values

$$
\begin{aligned}
& \mathrm{jj}=1 \text {. } \\
& \text { for }(\mathrm{jj}=0 ; \mathrm{jj}<10 ; \mathrm{jj}++) \\
& \{ \\
& \mathrm{t}=0 \text {; } \\
& \text { for(ii=1;ii<10;ii++) } \\
& \mathrm{t}=(\mathrm{t}+\mathrm{xx}[\mathrm{ii}]) \text {; } \\
& \text { for(ii=0;ii<10;ii++) } \\
& \text { \{ } \\
& \text { if(ii==9) } \\
& \mathrm{tt} 2[\mathrm{ii}]=\mathrm{t} \text {; } \\
& \text { else } \\
& \mathrm{tt} 2[\mathrm{i}]=\mathrm{xx}[\mathrm{ii}+1] \text {; } \\
& \} \\
& \mathrm{jj}=9 \text {; } \\
& \text { for }(\mathrm{ii}=0 ; \mathrm{ii}<10 ; \mathrm{ii}++) \\
& \{ \\
& \mathrm{xx}[\mathrm{ii}]=\mathrm{tt} 2[\mathrm{jj}] \text {; } \\
& \text { jj--; } \\
& \text { \} }
\end{aligned}
$$


3) The ten key generated in stage 2 are reduced to five that is first key is XOR with tenth key and the result is stored in a separate $\operatorname{array}(\mathrm{kk} 1)$ in first position after a left shift by zero, then second key XOR with ninth key and result is stored in array(kk1) second position after a left shift by one and the process continues till stage 2 ten keys are reduced to five. The process is shown below:

$$
\begin{gathered}
\mathrm{jj}=10 ; \\
\text { for }(\mathrm{ii}=0 ; \mathrm{ii}>\mathrm{jj} ; \mathrm{ii}++) \\
\{ \\
\mathrm{kk1} 1[\mathrm{ii}]=(\mathrm{kk} 1[\mathrm{ii}]+\mathrm{kk} 1[\mathrm{jj}])<<\mathrm{ii} ; \\
\mathrm{jj}=\mathrm{jj}-1 ; \\
\}
\end{gathered}
$$

4) The ten keys of stage 2 are reduced to five in stage 3 then in stage 4 this five keys are XOR and the result is stored in a variable $(\mathrm{kkmm})$. the output of stage 4 will be the input to stage5.

$$
\begin{aligned}
& \mathrm{kkmm}=0 \\
& \text { for }(\mathrm{ii}=0 ; \mathrm{ii}<5 ; \mathrm{ii}++) \\
& \mathrm{kkmm}=\left(\mathrm{kkmm}^{\wedge} \mathrm{kk} 1[\mathrm{ii}]\right) \text {; }
\end{aligned}
$$

5) Finally the result of stage4 is reduced to a number less than 10 by applying modular operation. This result as shown in Table II will be acting a key.

Shared secret key for session $=77$

TABLE II. GENERATED KEY

\begin{tabular}{|l|l|}
\hline KKi & Value \\
\hline KK1 & 1155 \\
\hline KK2 & 2156 \\
\hline KK3 & 4235 \\
\hline KK4 & 8316 \\
\hline KK5 & 16555 \\
\hline KK6 & 32956 \\
\hline KK7 & 65835 \\
\hline KK8 & 131516 \\
\hline KK9 & 262955 \\
\hline KK10 & 525756 \\
\hline
\end{tabular}

Final value of Key $=3.000000$

Step 2: Take the input message, for example " $24,32,56$, 65,73 ". Here every element of input message will be encrypted by calling the trigonohash technique. The input $(x x)$ is taken from input string to be encrypted, first the input is converted into radians by multiplying $x x$ with pi/180.0 and the generated value is given to Cos function. The output of Cos function will be the input to Sin function. The output of Sin function is again manipulated which forms the final cipher text to be transmitted to destination. So the process of encryption can be described as four step process as illustrated below:

$$
\begin{aligned}
& \text { for(ii=0;ii<nx;ii++) } / / n x \rightarrow \text { Number of input data } \\
& \{ \\
& \text { yy } 11[\mathrm{ii}]=\cos (\mathrm{sz}[\mathrm{i}] * \mathrm{key} / 180.0) ; / / \mathrm{sz}[\mathrm{i}] \rightarrow \mathrm{i}^{\text {th }} \text { Data } \\
& \text { yy12[ii] }=\sin (\mathrm{yy} 11[\mathrm{ii}] * \mathrm{key} / 180.0) \text {; } \\
& \text { ss1=int(yy12[ii]); } \\
& \text { frac1 }=\text { yy } 12[\text { ii] }]-s s 1 \text {; } \\
& \text { ss2 = Mod_Int3(ss1); } \\
& \text { yy } 12[\text { ii }]=s s 2 \text {; } \\
& \text { yy12[ii]=yy } 12[\text { ii }]+\text { frac } 1 \text {; } \\
& \text { ab1[ii]= hash1(sz[i]); } \\
& \text { \} }
\end{aligned}
$$

int Mod_Int3(int xx)

\{

int static r1;

int $\mathrm{AA} 1, \mathrm{BB} 1, \mathrm{AA} 2, \mathrm{BB} 2$;

$$
\begin{aligned}
& \mathrm{AA} 1=\mathrm{xx} \& 0 \mathrm{xff} ; \\
& \mathrm{BB} 1=(\mathrm{xx}>>8) \& 0 \mathrm{xff} ; \\
& \mathrm{AA} 2=(\mathrm{xx}>>16) \& 0 \mathrm{xff} ; \\
& \mathrm{BB} 2=(\mathrm{xx}>>24) \& 0 \mathrm{xff} ; \\
& \mathrm{r} 1=(\mathrm{AA} 1<<24)+(\mathrm{BB} 1<<16)+(\mathrm{AA} 2<<8)+(\mathrm{BB} 2) ;
\end{aligned}
$$

return $\mathrm{r} 1$;

\}

These values( $x x$ in radian) will go into the $\operatorname{Cos}(x x)$, and the output of Sin (yy) after manipulating it with Mod_Int3 function form the cipher text. As we have changed the value of $\pi=\mathrm{SK}_{1}=1.137201$ (for example) the result will be automatically changed as seen in the cipher text.

The general methodology for calculating cipher text is a four step process.

1) The first input is given to $\operatorname{Cos}($ input*key/180.0) as $\operatorname{Cos}$ function takes input in radian form, the input is multiplied with $\pi / 180$ here $\pi$ is assigned the value of generated key.

2) The output of $\operatorname{Cos}$ function will be the input to Sin function i.e. the out will be given to $\operatorname{Sin}(\cos$ output*key/180.0), as $\operatorname{Sin}$ also takes radian value the same procedure is adopted as above for generating output.

3) The output of Sin function is manipulated using Mod_Int3 function which rearranges the bytes in the input i.e in Sin function output least significant byte is placed most significant bytes place i.e interchanged then (least significant 1) byte is interchanged with (most significant -1) this is repeated for all the bytes $/ 2$ in the output. The output of 
Mod_Int3 is the final cipher text which is transmitted to destination along with the hash value(HA-160) of input.

Now if yy12[ii] and encryption algorithm are known to the attackers then also they will not be able to breach the information. Because the value of $\pi$ is not general like $\pi=3.141592$ rather than it is $1.137201\left(\mathrm{SK}_{1}\right.$ or generated key) Experimental results are shown in Table III.

TABLE III. DEMONSTRATION OF ENCRYPTION PROCESS

\begin{tabular}{|c|c|c|c|}
\hline $\begin{array}{l}\text { Input } \\
\text { Message }\end{array}$ & $\begin{array}{l}\text { Key } \\
\text { (pi) }\end{array}$ & CipherText & Hash value of Input \\
\hline 24 & \multirow{5}{*}{3.000000} & 0.015350 & $\begin{array}{l}\text { 05c8778d2274ca4940aca31e32a7 } \\
\text { 1ebfc99b597d }\end{array}$ \\
\hline 32 & & 0.014351 & $\begin{array}{l}\text { 82817f1da244ca39d0dca32eb277 } \\
\text { 1eaf4654610d }\end{array}$ \\
\hline 56 & & 0.009919 & $\begin{array}{l}\text { 2814db78a2388a55c37d1a4ab26a } \\
\text { decbebe7bd68 }\end{array}$ \\
\hline 65 & & 0.007806 & $\begin{array}{l}\text { 4f15dbe822088a4553ad1a5a323a } \\
\text { debb12e8bdd8 }\end{array}$ \\
\hline 73 & & 0.005780 & $\begin{array}{l}\text { e514db78a2388a55c37d1a4ab26a } \\
\text { decba8e7bd68 }\end{array}$ \\
\hline
\end{tabular}

\section{DECRYPTION PROCESS}

Take the input message, for example "0.000256, 0.000239, $0.000165,0.000130,0.000096$ ".Here every element of input message will be decrypted by calling the trigonohash technique. The $\operatorname{input}(x x)$ is taken from input string to be decrypted, first in the input, the effect of Mod_Int3 function of encryption module is nullified by arranging the bytes of input in original form by using Mod_Int3 function of decryption module. This output is given to Sin function and the output of $a \operatorname{Sin}$ function will go as input to $a C o s$ function. The output of aCos function is given to hash function(HA-160). The calculated hash code is matched with the received hash code, if matched then the output of aCos function is accepted as the message transmitted by sender else rejected. So the process of decryption can be described as four step process as illustrated below:

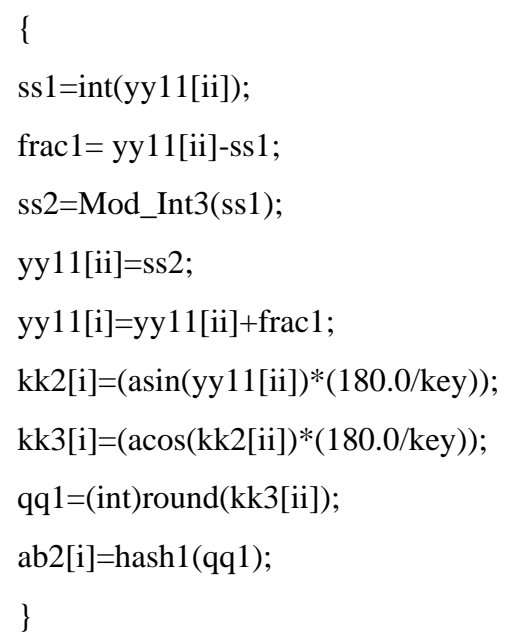

\section{\{}

int static $\mathrm{r} 1$;

int $\mathrm{AA} 1, \mathrm{BB} 1, \mathrm{AA} 2, \mathrm{BB} 2$;

AA1 $=x x \& 0 x f f$

$\mathrm{BB} 1=(\mathrm{xx}>>8) \& 0 \mathrm{xff}$

$\mathrm{AA} 2=(\mathrm{xx}>>16) \& 0 \mathrm{xff} ;$

$\mathrm{BB} 2=(\mathrm{xx}>>24) \& 0 \mathrm{xff}$;

$\mathrm{r} 1=(\mathrm{AA} 1<<24)+(\mathrm{BB} 1<<16)+(\mathrm{AA} 2<<8)+(\mathrm{BB} 2) ;$

return $\mathrm{r} 1$;

\}

These values $(x x)$ after rearrangement with Mod_Int3 function will go into the $\operatorname{aSin}(x x)$, and the output of $a \operatorname{Cos}$ (yy) after calculating the message digest of output and comparing it with received message digest, if the comparison is positive will form the plane text or message to be accepted by receiver. As we have changed the value of $\pi=\mathrm{SK}_{1}=$ 1.137201 (for example), the result will be automatically changed as seen in the plane text.

The general methodology for calculating plain text is a four step process:

1) The input to decryption algorithm is manipulated using Mod_Int3 function which rearranges the bytes in the input i.e input least significant byte is placed most significant bytes place that means interchanged then (least significant -1 ) byte is interchanged with (most significant -1) this is repeated for all the bytes $/ 2$ in the input. The output of Mod_Int3 is given as input to next stage.

2) The output of previous stage acts as the input to Sin function i.e. the out will be given to ( $a \operatorname{Sin}(\mathrm{aTan}$ output)*key/180.0), as aSin also takes radian value the same procedure is adopted as above for generating output.

3) The output of aSinfunction will be the input to $a \operatorname{Cos}$ function i.e. the output will be given to $(a \operatorname{Cos}(a \operatorname{Sin}$ output)*key/180.0), as aSinfunction also takes radian value the same procedure is adopted as above for generating output as above for generating output. The output of $a C o s$ is given to Hash function(HA-160) for generating message digest, then the generated message digest is compared with received message digest(from sender), if matched then only the output of $a C o s$ is accepted as received message(plain text) otherwise it is rejected. Experimental results are shown in Table IV.

TABLE IV. DEMONSTRATION OF DECRYPTION PROCESS

\begin{tabular}{|c|c|c|c|}
\hline Ciphertext & Key(pi) & $\begin{array}{l}\text { Hash value } \\
\text { check }\end{array}$ & Plain Text \\
\hline 0.015350 & 3.0000 & \multirow{5}{*}{$\begin{array}{l}\text { Hash value check } \\
\text { for each cipher } \\
\text { text }\end{array}$} & 24 \\
\hline 0.014351 & 3.0000 & & 32 \\
\hline 0.009919 & 3.0000 & & 56 \\
\hline 0.007806 & 3.0000 & & 65 \\
\hline 0.005780 & 3.0000 & & 73 \\
\hline
\end{tabular}




\section{MYSTERY KEY FOR SESSION UPDATE PHASE AND USER PASSWORD UPDATE PHASE}

Right when the data correspondence time among sender and beneficiary methodologies update period, GN Server makes information for update secret key for next session(DUMK) and encodes it and sends it to both User and Sensor Node.

\section{EIDUMKSs $=\mathrm{X}$ IDs - GN $\oplus$ DUMK \\ EIDUMKUm $=$ MM2 $\oplus$ DUMK}

At that point server sends EIDUMKSs (encrypted data for update mystery key for next(new) meeting) to the Sensor Node and EIDUMKUm (encrypted data for update mystery key for next(new) meeting) to the User. Subsequent to getting this data, Sensor Node quits sending information and hangs tight for another mystery key meeting. Subsequent to getting the solicitation both User and Sensor Node suspends their activity and User sends $\{\mathrm{IDm}, \mathrm{RPWm}, \sigma \mathrm{m}\}$ to GN Server and the cycle of enlistment and verification and meeting key synchronization start which brings about formation of new meeting key(i.e. second meeting so $\mathrm{SKS}_{2}$ ) at both at mobile device(user) and Sensor Node(Ss) additionally at this junction, if client needs he too can change his password(PWm).

The aggregate of the above are the nuances of the lightweight arrangement. The arrangement consolidates three phases. In like manner approval stage, we present a grouping key $\left(\mathrm{xx} 1, \mathrm{bm}, \mathrm{X}_{\mathrm{GN}}\right.$ and $\left.\mathrm{X}_{\mathrm{IDs}-\mathrm{GN}}\right)$ and pre realized a solitary bearing hash function(H1). It can comprehend shared approval just as hinder various attacks. Variable gathering key is proper for different applications and prevents attacks satisfactorily.

\section{DISCUSSION OF SECURITY FEATURES}

In this part, we show our protocol achieves some excellent functional properties and can resist well-known attacks. Besides, the comparative analysis of our protocol and other relevant protocols are also given in this part.

\section{A. Proper Mutual Affirmation}

The versatile device(user) and GN Server can validate each other in light of the fact that toward the beginning of verification, mobile device(user) himself checks if he is certified by contrasting a worth he makes i.e Am = A'm. The client registers A'm esteems from the information which he supplies at the hour of verification. The Am is pre figured worth done at the hour of enrollment from the data $(\mathrm{Bm})$ given by GN server. So whenever figured value $\left(\mathrm{A}^{\prime} \mathrm{m}\right)$ is equivalent to preexisted value(Am) which implies that client is certifiable and when GN server registers MM5 = MM'5, GN server gets a confirmation that the client is real Also the sensor hub get an affirmation about the GN server when it checks MM7=MM'7 . The GN server gets an affirmation about the sensor hub when it figures MM'8 and contrasts it and the got MM8 esteem from the sensor hub i.e MM'8=MM8 gives a confirmation to the GN server about the sensor hub. The portable device(user) get an affirmation about GN server when he figures MMM'10 and contrasts it and the got MMM10 esteem from the GN server. The GN server and mobile device(user) has a pre shared one way hash work $\mathrm{H} 1(\cdot)$, just real GN Server and portable device(user) realizes the arranged one way hash work H1(·) and the GN worker and sensor hub has a pre shared one way hash work $\mathrm{H} 1(\cdot)$, just real GN Server and sensor node(Ss) realizes the arranged one way hash work $\mathrm{H} 1(\cdot)$.

\section{B. Quickly Identification for Unapproved Login}

Here the plan the Sensor Node information can be gotten to by versatile device(user) simply in the wake of passing shared confirmation and key synchronization stage and toward the beginning of the stage the client's login(identity ID), secret word and biometric needs to pass the check. Some unacceptable secret key checking instrument permits the cell phone to rapidly recognize and dismiss unapproved login brought about by wrong secret word, and the confirmation cycle can be found in the Step 1 of shared verification and key synchronization stage. Nonetheless, the protocol [17] had no such instrument to recognize wrong secret word.

\section{Oppose Mobile Device Misfortune Attack}

In the event that an aggressor $\mathrm{A}$ gets Um's cell phone , $A$ can recover the parameters $\{B m, \operatorname{Gen}(),. \operatorname{Rep}(),. X X, \tau m\}$ in the cell phone by utilizing power examination attack[18]. Here $\mathrm{Bm}=\mathrm{Am} \oplus(\mathrm{RPWm} \oplus \sigma \mathrm{m}), \mathrm{RPWm}=(\mathrm{PWm} \bigoplus \mathrm{rm})$.

$\mathrm{Am}=\mathrm{Bm} \oplus(\mathrm{RPWm} \oplus \sigma \mathrm{m})$, and $\sigma \mathrm{m}$ is an irregular number extricated from Um's biometric information. As Bm, RPWm and Am are figured from obscure arbitrary numbers RPWm, rm , om and H1(one way hash work), the assaulter $A$ will be unable to figure these boundaries precisely which may bring about finish of validation stage as the variable A'm at the hour of verification may not match so our protocol can dodge cell phone misfortune assault.

\section{Customer Mystery and Intractability}

Believe that a login demand message (MM3, MM4,MM5 is snooped by aggressor $A$, where MM3= IDm $\bigoplus \mathrm{MM} 2$, MM4=IDs $\oplus \mathrm{MM} 2$ and $\mathrm{MM} 5=\mathrm{H} 1\left(\mathrm{~A}^{\prime} \mathrm{m}\right)$ where $\mathrm{MM} 2=$ $\mathrm{bm}^{*} \mathrm{XX}$ is critical and this key is the result of private key of user(bm) utilizing elliptic curve idea and $\mathrm{XX}$ is the public key of GN server utilizing elliptic curve idea i.e. key is made by Diffie-Hellman key exchange idea, assailant $A$ can't recover MM3,MM4 until it have the data about the private key of GN Server or private key of mobile device(user). MM5 is created from Am which is known to just mobile device(user) and GN server and from hash code the assaulter A can't recover Am. So by utilizing private key and public key (elliptic curve idea and Diffie-Hellman key exchange), symmetric mystery key of $\mathrm{GN}$ server $\left(\mathrm{X}_{\mathrm{GN}}\right)$ and one way hash work $\mathrm{H} 1(\cdot)$, the proposed convention accomplishes the component of client mystery. Here after GN Server gets the login data it can recover the Um recognizable proof (IDm) as it has the key additionally in this protocol first the client personality check is done carefully toward the beginning of validation and key synchronization phase $\left(\mathrm{Am}=\mathrm{A}^{\prime} \mathrm{m}\right)$. Likewise the component of login request message relies upon bm(user private key), in this way aggressor $A$ can't follow User(as no fundamental data is passed straightforwardly) from public channel. In any case, the scheme [17] can't accomplish the capacity of intractability.

\section{E. Sensor Hub Mystery}

In this plan, Sensor Node's personality isn't sent out in the open channel as plaintext. At the point when Um needs to get 
to the Sensor Node(Ss) information, Sn character is scrambled when Um send the solicitation to GN server for correspondence with Sensor Node Ss which GN Sensor can recover by decoding it with comparing secret key. Any aggressor without the information on relating secret word can't get Sensor Node's personality IDs and the plan accomplishes the component of sensor hub namelessness.

\section{F. Appropriate for Internet of Things Applications}

In our plan, GN Server is a confided in outsider for mobile device(user) and Sensor Node(Ss) and the verification and key synchronization stage is finished with the assistance of GN Server. With this the over-burden of both User and Sensor Node is diminished additionally it saves energy utilization of both portable device(user) and Sensor $\operatorname{Node}(\mathrm{Sn})$ (as the energy utilization of sensor hub is straightforwardly extent to the correspondence distance) also expands the life cycle of sensor nodes. Alongside when the common validation and key synchronization stage finishes, a meeting key SKS at both mobile device(user(Um)) and Sensor $\operatorname{Node}(\mathrm{Ss})$ is given by GN server, which can guarantee the resulting secure correspondence among Um and Ss. Consequently the portray design for our plan is reasonable for most IoTapplication. However, in some past related work [13], the client discuss straightforwardly with sensor hubs, it would lessen the existence pattern of sensor organizations and isn't reasonable for IoT applications.

\section{G. Restrict Impersonation Attack}

From the depiction of verification and meeting key Agreement stage, assailant $A$ necessities the data of IDm and $\mathrm{Am}=\left(\mathrm{IDm} \oplus \mathrm{X}_{\mathrm{GN}}\right)$ to mimic as a real client to make a substantial login demand. However, from the description above, the proposed protocol provides the feature of user anonymity, and any gathering with the exception of GN can't get client's personality IDm. Likewise, Am must be recovered by Um from Bm utilizing $\mathrm{PWm}, \mathrm{rm}$ and $\sigma \mathrm{m}$ or can be determined by GN server utilizing IDm and GNs(secret key of GN server) when he/she gets IDm. Therefore, aggressor $A$ can't get Am without knowing required data, and our protocol can maintain a strategic distance from client impersonation assault. Additionally, to mirror as the GN worker, the private keys $\mathrm{xx} 1$ and GNs(secret key of server) is fundamental data for assailant $A$ to produce substantial correspondence messages. Nonetheless, $\mathrm{xx} 1$ (private key) and mystery $\operatorname{key}\left(\mathrm{X}_{\mathrm{GN}}\right)$ of $\mathrm{GN}$ server and known to GN server just, and the proposed convention can stay away from entryway impersonation assault.

\section{H. Oppose Replay Assault}

In this plan, the single direction hash work system is received to oppose replay assault. In various strides of confirmation and key synchronization period of the plan, the hash code is created by Um, GN Server and Sensor Node for processing correspondence messages. Since the hash code carefully follow avalanche effect little change in message will create gigantic change in hash code so it would be hard for an assaulter $A$ to figure the shared message on which the hash code are produced, so it is hard to perform replay assault. Consequently, our plan is secure from replay assault.

\section{Simple Secret Key Change}

In our plan, as the update time frame for key finishes. The GN worker starts a solicitation for change of mystery key for meeting. For this the GN server sends encoded message to User and sensor hub, so the cycle of progress of secret phrase begins. In the wake of accepting the solicitation both mobile device(user) and Sensor Node(Ss) suspends their activity and User sends $\{$ IDm , RPWm, $\sigma \mathrm{m}\}$ to GN Server and the cycle of enlistment and confirmation and meeting key synchronization start which brings about production of new meeting both at User and Sensor Node. At this intersection the mobile device (user) can likewise change his password (PWm).

TABLE V. PARAMETERS VALUES

Parameters Values(in bits)
IDm - 80
IDs - 160
$\mathrm{X}_{\mathrm{GN}}, \mathrm{X}_{\mathrm{IDs} \text {-GN }} \mathrm{PWm}$ (All Password) - 160
bm,Xx1,XX,MM1 - 320
Hash value - 160
Note: Values taken as per Xiong-Li[21]

\section{PERformance ANALySIS}

\section{A. Process Computation Time}

We look at the time of the common attestation stage, which is executed using[19][20] in the three conventions (DES algorithm, Xiong -li scheme(2017) and our convention). Analysis, were led on a PC with windows7 32bit, $2.00 \mathrm{GHz}$ with $2 \mathrm{~GB}$ of internal memory utilizing $\mathrm{C} / \mathrm{C}++$ language. The yield says that our plan accomplishes the best time execution of $31000 \mu \mathrm{s}$ (microseconds) as shown in Fig. 8, DES algorithm $31000 \mu \mathrm{s}$ (microseconds) and Xiong-Li(2017) common attestation time is $31000 \mu \mathrm{s}$ (microseconds), which is shown in the Fig. 9 beneath.

Our proposed plot is having less overhead, and can restrict various attacks. Researching Xiong-Li(2017)[21] basic affirmation in this arrangement it allows the sensor device to go about as server as it makes the mysterious expression and accommodates customer and does an incredible arrangement getting ready which is illicit of lightweight count rules. Also ChangLi(P1) plan like shared confirmation isn't done suitably, faces stolen smart card attack, further more encounters tracking attack, not applicable to practical application, delayed and costly detection of wrong password input, lack of identity detection mechanism and unfriendly mystery key change In our arrangement, we simply use XOR and XNOR action, one way Hash work and elliptic curve cryptography methodology for shared approval and meeting key synchronization. Moreover, session key is created by server and synchronized with IoT node and user (mobile device). Fig. 9 shows that our scheme takes minimum time for authentication. It is a nice technique to minimize the overhead of IoT hub. 


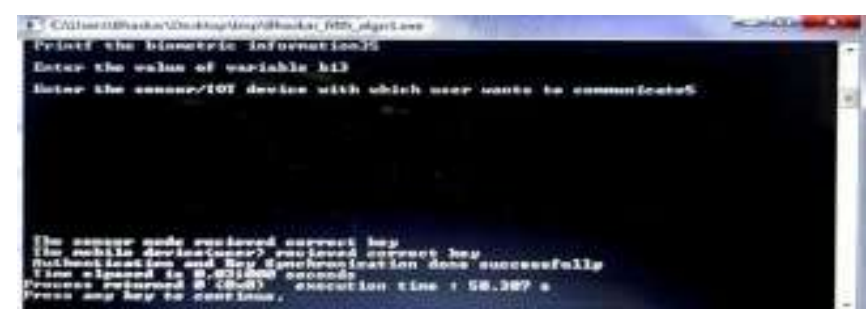

Fig. 8. Result of Execution of Our Algorithm.

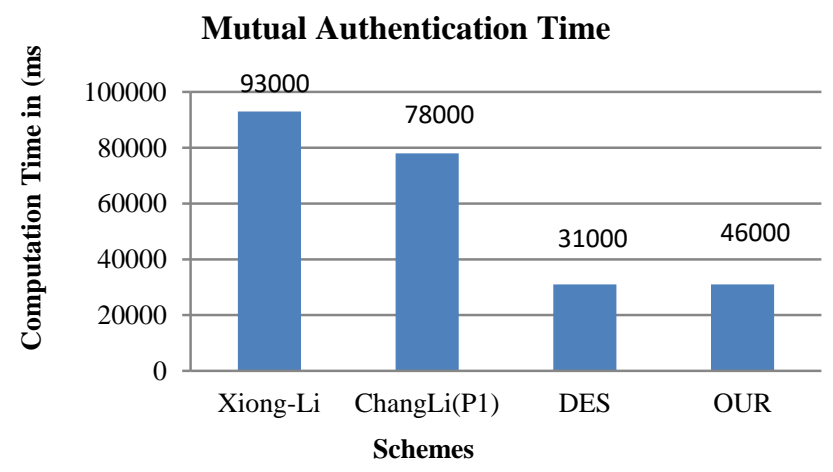

Fig. 9. Examination of Shared Confirmation Time among Xiong -Li(2017), Our Scheme and DES Algorithm.

\section{B. Performance Analysis of Hash Algorithm(HA-160)}

These three calculations HA-160, SHA1 and MD5 were tried for comparison dependent on the execution time necessities as shown in Fig. 10, Fig. 11 and Fig. 12. All the calculations have been actualized in $\mathrm{C} / \mathrm{C}++$ and run on windows 7 32-bit, CPU $2.00 \mathrm{GHz}$ with $2 \mathrm{~GB}$ of internal memory. With the aftereffects of the analysis, it was discovered that SHA1 and MD5 requests more execution time than HA160 to create hash code.

The outcome in above Fig.11 shows time taken by three calculation (MD5, SHA1, and HA160(size of message taken by HA160 is double than SHA1)) for producing hash code for the message " The quick brown fox jumped on the lazy dog ".

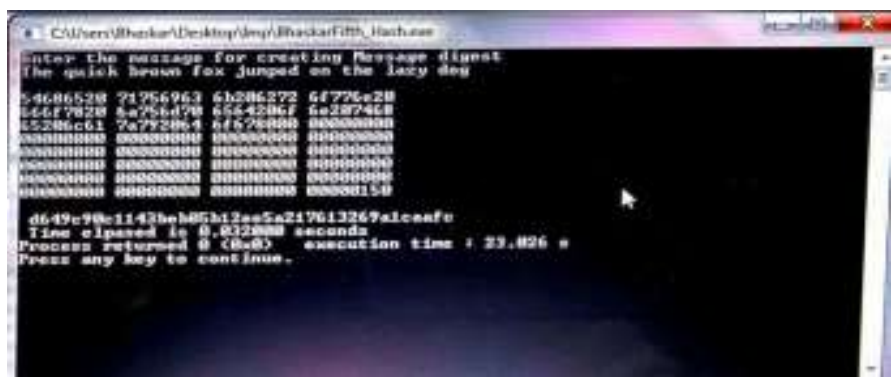

Fig. 10. Processing Time by HA-160 Algorithm"The Quick Brown Fox Jumped on the Lazy Dog".

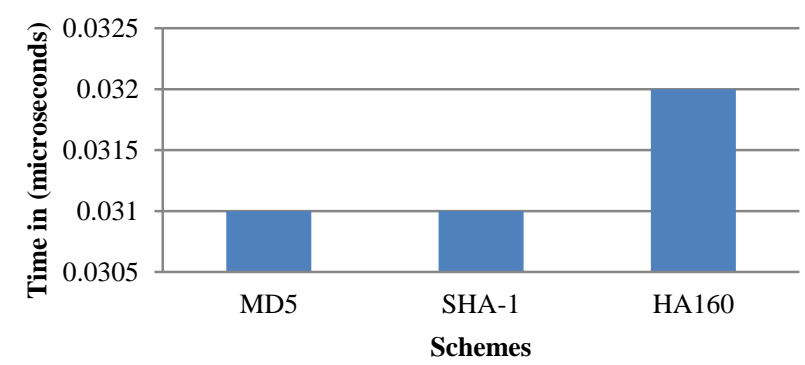

Fig. 11. Assessment of MD5, SHA1 and HA 160 Concerning Time Taken to Execute the Message "The Quick Brown Fox Jumped on the lazy Dog "i.e. 34 Bytes.

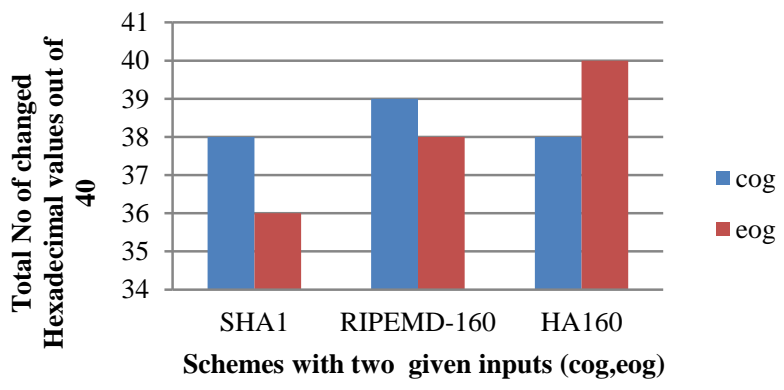

Fig. 12. Demonstrating Avalanche Effect. The Input Message used and the Two Altered Message (Dog is Replaced by Cog and Eog in Input Message) for Comparison is Shown Below.
A. Message: "The quick brown fox jumped on the lazy dog " Compared with
$\operatorname{cog}$ :"The quick brown fox jumped on the lazy cog "
eog:"The quick brown fox jumped on the lazy eog "

Table VI shows the message digest conveyed by three calculation (HA160, SHA-1 and RIPEMD160) for the message "The quick brown fox jumped on the lazy dog". Table VII shows the adjustment in hash code when the message in Table VI is modified for example dog is dislodged with $\operatorname{cog}$, HA-160 produces a hash code with change of paying little heed to hexadecimal attributes from $\mathrm{e}$ and f(starting from left showed up in solid), SHA-1 yield is a hash code with change of with or without hexadecimal attributes from 9 and 1(starting from left showed up in serious) and RIPEMD 160 produces a message digest with change of paying little mind to hexadecimal attributes from $\mathrm{f}$ (starting from left showed up in strong) and Table VIII shows the adjustment in hash code when the message in Table VI is adapted to occurrence dog is supplanted with eog, HA-160 produces a message digest with change of each hexadecimal quality, SHA-1 yield is a hash code with change of with or without hexadecimal attributes from f, 2, 4 and 3(starting from left showed up in unprecedented) and RIPEMD 160 produces a message digest with change of with or without hexadecimal qualities from 4 and 4(starting from left showed up in strong).So on an ordinary HA-160 shows better result when any character is changed. The result is better than SHA160 and RIPEMD160 as shown in Fig. 11. 
TABLE VI. RESULT

Algo/Input: "The quick brown fox jumped on the lazy dog"

HA160: a5abc142 e821f1a2 48d60829 f8544618 697ea332

SHA1: 42914f6d 22976baf d4bab416 fe23b03d 4fa74963

RIPEMD160 :39349b80 fc87ec4c 887ccd87 4943f253 ee051126

TABLE VII. RESULT (IN INPUT DOG CHANGED TO COG)

Algo/Input: "The quick brown fox jumped on the lazycog"

HA160: 58713d84 ebe7200f 24c245a4 fc197485 1c441f74

SHA1 :99e9c9dc 4698e297 efe02e13 dc3d7e97 f5355001

RIPEMD160:5fb682d2 4aa561f7 070774db 001cf3d6 dbdb7061

TABLE VIII. RESULT (IN INPUT DOG CHANGED TO EOG)

Algo/Input: "The quick brown fox jumped on the lazyeog"

HA160: 04b36898 26446645 7659cb26 3676babb c8864a88

SHA1 :7f036e54 7b914f0f 3cadc54d 572cfa6b eb634b13

RIPEMD160:ac46cdbc 2ff1300a 1eeff31a 4f4f956c dfdb483f

\section{Communication Overhead}

To investigate the correspondence overhead, we expect that the eight messages (i.e., Message1, Message2, Message3, Message4, Message5, Message6, Message7 and Message8) are communicated during the enrollment system and the confirmation method. All the more accurately, Message1 and Message 2 are sent in the enlistment strategy and Message3, Message4, Message5, Message6, Message7 and Message8 are communicated in the verification technique. Message1 incorporates the sensor's character IDm, RPWm(encrypted password) and uniform random number generated from Biometric information $\left(\sigma_{m}\right)$ and Message-2 includes $\mathrm{Bm}((\mathrm{a}$ value generate from GWN server from Am) and XX(public key of GWN Server) . In addition, Message3 includes MM1, MM3, MM4 and MM5 which are calculated as follows:

$|\mathrm{MM} 1|=|\mathrm{bm} * \mathrm{PP}|$

|MM3|=| MM2 $\oplus \mathrm{IDm} \mid$

|MM4|=| MM2 $\oplus$ IDs $)$ |

$|\mathrm{MM} 5|=\left|\mathrm{H} 1\left(\mathrm{~A}^{\prime} \mathrm{m}\right)\right|$

Moreover, the parameters MM6 and MM7 in Message 4, are calculated as follows:

$|\mathrm{MM} 6|=\left|\mathrm{X}_{\text {IDs -GN }}^{\prime} \bigoplus \mathrm{ID}^{\prime} \mathrm{m}\right|$

$|\mathrm{MM} 7|=\left|\mathrm{H} 1\left(\mathrm{X}_{\text {IDs -GN }}^{\prime}\right)\right|$

then, the parameter MM8 in Message 5, is calculated as follows:

MM8 =H1( $\mathrm{X}_{\text {IDs -GN }} \bigoplus$ ID"m)

also, the parameter MM9, MM10 and MMM10 in Message 6 , is calculated as follows:

$|\mathrm{MM} 9|=\left|\mathrm{X}_{\mathrm{IDs}-\mathrm{GN}}^{\prime} \oplus \mathrm{SKS}\right|$

$|\mathrm{MM} 10|=|\mathrm{MM} 2 \oplus \mathrm{SKS}|$

$\mid$ MMM10 $|=| \mathrm{H} 1(\mathrm{~A} " \mathrm{~m} \oplus \mathrm{ID} ' \mathrm{~m}) \mid$

Finally, the parameter MM11 in Message 7 and MM12 in Message 8 is calculated as follows:
|MM11|=| H1(MM10)

|MM12|= |H1(MM9)|

Table $\mathrm{V}$ contains the setting of boundaries that we have accepted for assessing the correspondence overhead of the proposed component as shown in Table IX. Subsequently, the general transmission capacity overhead of the proposed component is determined as follows:

8

$$
\begin{gathered}
\text { bw }=\sum \text { Message } \mathrm{i} \\
\mathrm{i}=1
\end{gathered}
$$

Message $1=|\mathrm{IDm}|+|\mathrm{RPWm}|+\mid \sigma_{m}$ (random rumber $) \mid$ $=400$ bits

Message $2=|\mathrm{Bm}|+|\mathrm{XX}|=480$ bits

Message $3=\mid$ MM1 $|+|$ MM3 $|+|$ MM4 $|+|$ MM5 $\mid=$ 1120 bits

Message $4=\mid$ MM6 $|+|$ MM7 $\mid=320$ bits

Message $5=|\mathrm{MM} 8|=160$ bits

Message $6=\mid$ MM9 $|+|$ MM10 $|+|$ MMM10 $\mid=480$ bits

Message $7=\mid$ MM11 $\mid=160$ bits

Message $8=\mid$ MM12 $\mid=160$ bits

TABLE IX. COMMUNICATION COST OF OUR PROPOSED MECHANISM

\begin{tabular}{|l|l|}
\hline Scheme & Communication Cost \\
\hline Our Scheme & 3136 bits \\
\hline Xiong-Li[21] & 2688 bits \\
\hline Chang-Le's[21] & 2400 bits \\
\hline
\end{tabular}

\section{Computational Expense}

To figure the computational expense of the proposed system, we have thought about the accompanying documentations: Th indicates the expense of single direction hash work, Tm signifies the expense of Multiplication activity, Ts means the expense of encryption and unscrambling activity. In view of the three parts (i.e. brilliant sensor, client and verification worker) which are utilized in the proposed instrument, the computational expense of every segment is introduced as follows:

1) Smart Sensor: The brilliant sensor performs calculations just in the verification method.

2) User: The client performs calculations in the enlistment and verification stage.

3) GN Server: The GN server performs calculation activities in enlistment and validation stage. The proposed component's computational expense is delineated in Table X. Because of the way that the proposed verification system depends just on scalar duplication activity utilizing ECC, a symmetric encryption/decoding activity and hash activity.

We use the simulation result [23] where Tm(cost based only on scalar multiplication operation using ECC) $=1.226 \mathrm{~ms}$, Ts $($ cost based only on symmetric encryption/decryption operation $)=2.049 \mu$ s and $\mathrm{Th}($ cost based only on hash operation) $=2.580 \mu \mathrm{s}$. 
TABLE X. COMPUTATIONAL COST OF OUR Proposed MECHANISM

\begin{tabular}{|l|l|l|l|l|}
\hline Scheme & $\begin{array}{l}\text { User(Mobile } \\
\text { device) }\end{array}$ & $\begin{array}{l}\text { GWN } \\
\text { Server }\end{array}$ & $\begin{array}{l}\text { Smart } \\
\text { Sensor }\end{array}$ & Total Cost \\
\hline $\begin{array}{l}\text { Our } \\
\text { Scheme }\end{array}$ & $3 * \mathrm{Th}+2 * \mathrm{Tm}$ & $\begin{array}{l}6 * \mathrm{Th}+ \\
1 * \mathrm{Tm}\end{array}$ & $3 * \mathrm{Th}$ & $2482.96 \mu \mathrm{s}$ \\
\hline $\begin{array}{l}\text { Xiong- } \\
\text { Li[21] }\end{array}$ & $\begin{array}{l}7 * \mathrm{Th}+2 * \mathrm{Tm}+ \\
2 \mathrm{Ts}\end{array}$ & $\begin{array}{l}8 * \mathrm{Th}+ \\
1 * \mathrm{Tm}+ \\
4 \mathrm{Ts}\end{array}$ & $\begin{array}{l}4 * \mathrm{Th}+ \\
2 \mathrm{Ts}\end{array}$ & $3743.412 \mu \mathrm{s}$ \\
\hline $\begin{array}{l}\text { Chang- } \\
\text { Le's[21] }\end{array}$ & $6 * \mathrm{Th}+2 * \mathrm{Tm}$ & $8 * \mathrm{Th}$ & $\begin{array}{l}5 * \mathrm{Th} \\
+2 * \mathrm{Tm}\end{array}$ & $4953.000 \mu \mathrm{s}$ \\
\hline
\end{tabular}

Note: Considering the process computation time for mutual authentication, computation cost, communication cost and other indicators, our algorithm performs well in most of the cases. The most prominent advantage is its process computation time for mutual authentication and communication cost.

\section{E. Encryption and Decryption Algorithm Analysis}

The investigation of the proposed trigonohash calculation for encryption and unscrambling has been done and exhibited in Table III and Table IV. The Encryption and Decryption Algorithm was coded in $\mathrm{C} / \mathrm{C}++$ Language[19][20]. It was compiled with MinGW-GCC 4.8.1, on the Core 2 Duo Processor, $2.00 \mathrm{GHz}$ under windows $7 \mathrm{OS}(32$ bit). The investigation boundaries are plain content size (in Bytes) and time taken in encryption and unscrambling (in $\mu$ seconds).

a) Comparative PerformanceAnalysis: The proposed Trigonohash algorithm(LW-algorithm) has been compared with other existed algorithms like RC4, Hill-Cipher[19][20], RSA, Present(Block Cipher with key 80 bits and plain content 64 bits or 8 bytes) [27] and ELSCA(LW-Stream Cipher) (Varient-HassanNoura(2019(key consist of 4 location)) stream cipher )[22]. Likewise, the results are shown below. From the going with Fig. 13 and Table XI we can say that the run time unpredictability of trigonometric Algorithm is underneath than the other existed Algorithm plot.

TABLE XI. SAMPLE RESUlt FROM DifFERENT ALGORITHM.(RESUlt IN MICROSECOND)

\begin{tabular}{|c|c|c|c|c|c|c|}
\hline $\begin{array}{l}\text { No } \\
\text { of } \\
\text { By } \\
\text { tes }\end{array}$ & $\begin{array}{l}\mathrm{RC} 4 \\
\text { (strea } \\
\mathrm{m} \\
\text { cipher } \\
\text { ) }\end{array}$ & $\begin{array}{l}\text { Hill- } \\
\text { Cipher } \\
\text { (Symm } \\
\text { etric } \\
\text { encrypt } \\
\text { ion) }\end{array}$ & $\begin{array}{l}\text { RSA } \\
\text { (public } \\
\text { key } \\
\text { encrypt } \\
\text { ion) }\end{array}$ & $\begin{array}{l}\text { Present(L } \\
\text { W-Block } \\
\text { Cipher(6 } \\
4 \text { bits)) }\end{array}$ & $\begin{array}{l}\text { ELSCA(L } \\
\text { WStreamCi } \\
\text { pher-2017) }\end{array}$ & $\begin{array}{l}\text { Trignohas } \\
\mathrm{h}(\mathrm{LW}- \\
\text { algorithm } \\
\text { ) }\end{array}$ \\
\hline 12 & 31000 & 16000 & 31000 & 47000 & 15000 & 00000 \\
\hline 20 & 46000 & 46000 & 31000 & 78000 & 46000 & 16000 \\
\hline 28 & 62000 & 63000 & 47000 & 125000 & 47000 & 31000 \\
\hline 36 & 93000 & 78000 & 78000 & 156000 & 93000 & 62000 \\
\hline
\end{tabular}

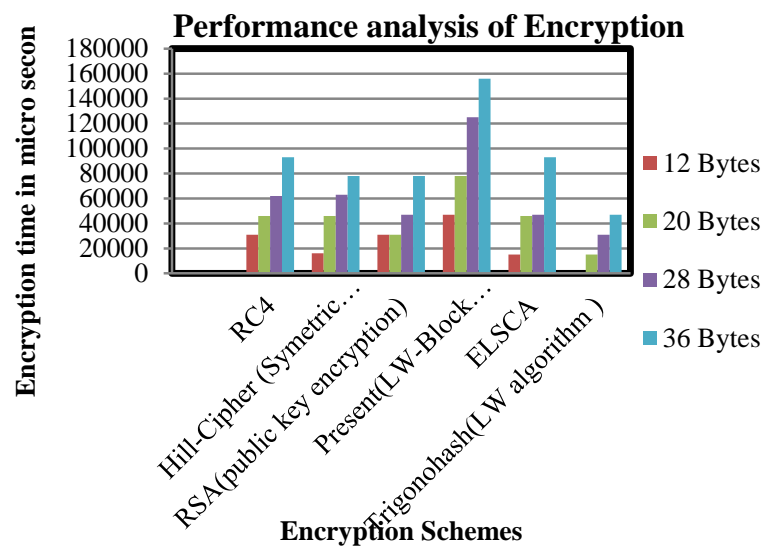

Fig. 13. Execution Examination of Encryption Algorithm of different Protocol.

\section{CONCLUSION}

In this paper, we propose a strong and weighty cryptographic arrangement for Industrial Internet of Thing using one way hash work, elliptic curve cryptography and trigonohash(trigonometry and hash work) idea and endeavored to improved security and execution of a cryptographic arrangement for lightweight contraption using key generation. It gives keen and feasible instruments for basic affirmation, meeting key synchronization and meeting key update. They are proper for IoT hub with limited handling resources and force. First thing, we present the nuances of shared affirmation using elliptic curve cryptography and one way hash work and a way to deal with hinder replay attack without timestamp. Besides, again elliptic curve cryptography and one way hash idea is progressed to simply recognize meeting key synchronization lastly by using mathematical thoughts data encryption and unscrambling are done to diminish the overhead of IoT center points. In addition, we have taken a gander at the security and execution of our arrangement with some lightweight approval and cryptographic plans. The arrangement is lightweight anyway can thwart attacks satisfactorily, which is useful for guaranteeing the security of the correspondence between IoT center points, users(mobile gadget) and server.

The proposed figuring for encryption isn't simply giving the quick data encryption anyway it gives an unrivaled security diverged from other count through a most grounded key as well. The assessment of $\pi$ is changing each time which is created from the common key that makes the count adequate. The estimation moreover makes the cryptanalysis cycle complex. Since there are dark variables will be more and the amount of conditions will be less when stood out from other standard encryption computations. This calculation could be well valuable for online applications like secure web based talking, data transmission between resource constraint devices and health sector. 


\section{REFERENCES}

[1] L. Atzori, A. Iera, and G. orabito, "The Internet of Things: A survey," Computer Networks, vol. 54, no. 15, pp. 2787 - 2805, 2010.

[2] L. Da Xu, W. He, and S. Li, "Internet of things in industries: A survey," IEEE Transactions on industrial informatics, vol. 10, no. 4, pp. 22332243, 2014.

[3] M. S. Hossain and G. Muhammad, "Cloud-assisted industrial internet of things (iiot)-enabled framework for health monitoring," Computer Networks, vol. 101, pp. 192-202, 2016.

[4] R. Amin, S. H. Islam, G. Biswas, M. K. Khan, L. Leng, and N. Kumar, "Design of an anonymity-preserving three-factor authenticated key exchange protocol for wireless sensor networks," Computer Networks, vol. 101, pp. 42-62, 2016.

[5] S. Sicari, A. Rizzardi, L. A. Grieco, and A. Coen-Porisini, "Security, privacy and trust in internet of things:The road ahead," Computer Networks, vol. 76, pp. 146-164, 2015.

[6] M. L. Das, "Two-factor user authentication in wireless sensor networks," IEEE Transactions on Wireless Communications, vol. 8, no. 3, pp. 1086-1090, 2009.

[7] A. K. Das, P. Sharma, S. Chatterjee, and J. K. singh, "A dynamic password-based user authentication scheme for hierarchical wireless sensor networks," Journal of Network and Computer Applications, vol. 35, no. 5, pp. 1646-1656, 2012.

[8] D. Wang and P. Wang, "Understanding security failures of two-factor authentication schemes for real-time applications in hierarchical wireless sensor networks," Ad Hoc Networks, vol. 20, pp. 1-15, 2014.

[9] K. Xue, C. Ma, P. Hong, and R. Ding, "A temporal- credential-based mutual authentication and key agree- ment scheme for wireless sensor networks," Journal of Network and Computer Applications, vol. 36, no. 1, pp. 316-323,2013.

[10] Q. Jiang, J. Ma, X. Lu, and Y. Tian, "An efficient two- factor user authentication scheme with unlinkabilityforwireless sensor networks," Peer-to-peer Networking and Applications,vol.8,no.6,pp.1070$1081,2015$.

[11] H.-L. Yeh, T.-H.Chen, P.-C.Liu, T.-H. Kim, and H.- W. Wei, “A secured authentication protocol for wireless sensor networks using elliptic curves cryptography," Sensors, vol. 11, no. 5, pp. 4767-4779, 2011.

[12] W. Shi and P. Gong, "A new user authentication protocol for wireless sensor networks using elliptic curves cryptography," International Journal of Distributed Sensor Networks, vol. 2013, Article ID 730831, 7 pageshttp://dx.doi.org/10.1155/2013/7308312013.

[13] Q. Jiang, J. Ma, F. Wei, Y. Tian, J. Shen, and Y. Yang, “An untraceable temporal-credential-based two-factor authentication scheme using ecc for wireless sensor networks," Journal of Network and Computer Applications, vol. 76, pp. 37-48, 2016.

[14] X. Li, J. Niu, S. Kumari, F. Wu, A. K. Sangaiah, and K.-K. R. Choo, "A three-factor anonymous /authentication scheme for wireless sensor networks in I nternet of things environments," Journal of Network and Computer Applications, 2017, doi:10.1016/j.jnca.2017.07.001.
[15] X. Li, J. Niu, M. Z. A. Bhuiyan, F. Wu, M. Karuppiah, and S. Kumari, "A robust ecc based provable secure authentication protocol with privacy protection for industrial internet of things," IEEE Transactions on Industrial Informatics, vol. PP, no. 99, pp. 1-1, 2017.

[16] Y. Dodis, L. Reyzin, and A. Smith, "Fuzzy extractors: How to generate strong keys from biometrics and other noisy data," in International Conference on the Theory and Applications of Cryptographic Techniques. Springer, 2004, pp. 523-540.

[17] C.-C. Chang and H.-D. Le, "A provably secure, efficient, and flexible authentication scheme for ad hoc wireless sensor networks," IEEE Transactions on Wireless Communications, vol. 15, no. 1, pp. 357-366, 2016.

[18] T. S. Messerges, E. A. Dabbish, and R. H. Sloan, "Examining smartcard security under the threat of power analysis attacks," IEEE Transactions on Computers, vol. 51, no. 5, pp. 541-552, 2002.

[19] 3GPP TS 35. 201 V14. 0. 0, Specificatio n of the 3GPP Confidentiality and Integrity Algorithms; Document 1: f8 and f9 specifications, 2017.

[20] 3GPP TS 35. 202 V14. 0. 0, Specificatio $\mathrm{n}$ of the 3GPP Confidentiality and Integrity Algorithms; Document 2: Kasumialgorithm specifications, 2017.

[21] Xiong Li, J Peng, J Niu, F Wu, J Liao, Kim Kwang, R Choo, “A Robust and Energy Efficient Authentication Protocol for Industrial Internet of Things" DOI 10.1109/JIOT.2017 2327-4662 (c) 2017 IEEE.

[22] Hassan Noura and Ali Chehab“ An Efficient and Secure Variant of RC4 Stream Cipher Scheme for Emerging Networks” 978-1-5386-56570/18/\$31.00_c 2019 IEEE.

[23] D. Wang, D. He, P. Wang, and C.-H. Chu, "Anonymous two-factor authentication in distributed systems: certain goals are beyond attainment," IEEE Transactions on Dependable and Secure Computing, vol. 12, no. 4, pp. 428-442, 2015.

[24] VenkateswaraRaoPallipamu, K Thammi Reddy, P Suresh Varma "ASH-160: A novel algorithm for secure hashing using geometric concepts" http://dx.doi.org/10.1016/j.jisa.2014.05.0012214-2126/@ 2014 Elsevier.

[25] VenkateswaraRaoPallipamu, K Thammi Reddy, P Suresh Varma "ASH512: Design and implementation of cryptographic hash algorithm using co-ordinate geometry concepts" http://dx.doi.org/10.1016/j.jisa.2014. 10.006, 2214-2126/C 2014 Elsevier.

[26] VenkateswaraRaoPallipamu, K Thammi Reddy, P Suresh Varma "Design and implementation of geometric based cryptographic hash algorithm: ASH-256" pp 275-291 ,[Rao* et al., 5(7): July, 2016] ICTM Value: 3.00 2016 IJESRT.

[27] A. Bogdanov, L. R. Knudsen, G. Leander, C. Paar, A. Poschmann, M. J.Robshaw, Y. Seurin, and C. Vikkelsoe, "Present: An ultra-lightweight block cipher," in International Workshop on CryptographicHardware and Embedded Systems. Springer, 2007, pp. 450-466.

[28] W.B. Heinzelman, A.P. Chandrakasan, H. Balakrishnan, An applicationspecific protocol architecture for wireless microsensor networks, IEEE Trans. Wirel. Commun. 1 (4) (2002) 660-670. 